\title{
Geometric modeling of some engineering GBT-Bézier surfaces with shape parameters and their applications
}

\author{
Sidra Maqsood ${ }^{1}$, Muhammad Abbas $^{1 *}$ (D), Kenjiro T. Miura ${ }^{2 *}$ (D), Abdul Majeed ${ }^{3} \mathbb{D}$, Samia BiBi ${ }^{4}$ and \\ Tahir Nazir ${ }^{1}$
}

\author{
"Correspondence: \\ muhammad.abbas@uos.edu.pk; \\ miura.kenjiro@shizuoka.ac.jp \\ 'Department of Mathematics, \\ University of Sargodha, Sargodha \\ 40100, Pakistan \\ ${ }^{2}$ Department of Mechanical \\ Engineering, Shizuoka University, \\ Hamamatsu, 432-8561, Shizuoka, \\ Japan \\ Full list of author information is \\ available at the end of the article
}

\begin{abstract}
This study is based on some $C^{1}, C^{2}$, and $C^{3}$ continuous computer-based surfaces that are modeled by using generalized blended trigonometric Bézier (shortly, GBT-Bézier) curves with shape parameters. Initially, generalized blended trigonometric Bernstein-like (shortly, GBTB) basis functions with two shape parameters are derived in explicit expression which satisfied the basic geometric features of the traditional Bernstein basis functions. Moreover, the GBT-Bézier curves and tensor product GBT-Bézier surfaces with two shape parameters are also presented. All geometric features of the proposed GBT-Bézier curves and surfaces are similar to the traditional Bézier curves and surfaces, but the shape-adjustment is the additional feature that the traditional Bézier curves and surfaces do not hold. Finally, a class of some complex computer-based engineering surfaces via GBT-Bézier curves with shape parameters is presented. In addition, two adjacent GBT-Bézier surfaces segments are connected by higher $C^{2}$ and $C^{3}$ continuity constraints than the existing only $C^{1}$ shape adjustable Bézier surfaces. Some practical examples are provided to show the efficiency of the proposed scheme and to prove it as another powerful way for the construction and modeling of various complex composite computer-based engineering surfaces using higher-order continuities.
\end{abstract}

Keywords: Generalized blended trigonometric Bernstein-like basis; Shape parameters; GBT-Bézier curves; Parametric continuity; GBT-Bézier surfaces; Computer-based engineering surfaces

\section{Springer}

\section{Introduction}

Bézier curves and surfaces techniques are very popular in computer technology, computer graphics (CG), and computer-aided geometric design (CAGD) due to their shape control parameters. In computer-aided manufacturing (CAM) and computer-aided design (CAD), Bézier curves and surfaces are robust tools for constructing free form curves and surfaces. Bézier curves and surfaces have an abundance of appealing in the fields of engineering, science, communications, and technology particularly in networks, animation, railway route, highway design, environment design, CAD system, and many other disciplines.

(c) The Author(s) 2021. This article is licensed under a Creative Commons Attribution 4.0 International License, which permits use sharing, adaptation, distribution and reproduction in any medium or format, as long as you give appropriate credit to the original author(s) and the source, provide a link to the Creative Commons licence, and indicate if changes were made. The images or other third party material in this article are included in the article's Creative Commons licence, unless indicated otherwise in a credit line to the material. If material is not included in the article's Creative Commons licence and your intended use is not permitted by statutory regulation or exceeds the permitted use, you will need to obtain permission directly from the copyright holder. To view a copy of this licence, visit http://creativecommons.org/licenses/by/4.0/. 
Table 1 Comparison of research work

\begin{tabular}{|c|c|c|}
\hline $\begin{array}{l}\text { Sr. } \\
\text { no }\end{array}$ & Existing method [11] & Proposed method \\
\hline 1 & $\begin{array}{l}\text { Hu et al. presented the SG-Bernstein basis functions } \\
\text { of order } n \text { with multiple shape parameters. }\end{array}$ & $\begin{array}{l}\text { The GBTB functions are constructed with only two } \\
\text { shape parameters. }\end{array}$ \\
\hline 2 & $\begin{array}{l}\text { Only simple SG-Bézier general cylinder has been } \\
\text { constructed. }\end{array}$ & $\begin{array}{l}\text { Two GBT-Bézier general cylinders can be connected } \\
\text { by higher } C^{2} \text { continuity smoothness. }\end{array}$ \\
\hline 3 & $\begin{array}{l}\text { Two SG-Bézier ruled surface patches have been } \\
\text { connected by only } C^{1} \text { continuity. }\end{array}$ & $\begin{array}{l}\text { Two GBT-Bézier ruled surfaces can be connected by } \\
\text { higher } C^{k}(k=2,3) \text { continuity constraints. }\end{array}$ \\
\hline 4 & $\begin{array}{l}\text { Two adjacent SG-Bézier swung surface patches } \\
\text { have been connected by only } \mathrm{C}^{1} \text { continuity. }\end{array}$ & $\begin{array}{l}\text { Two adjacent GBT-Bézier swung surfaces can be } \\
\text { connected by higher } C^{2} \text { continuity conditions. }\end{array}$ \\
\hline 5 & $\begin{array}{l}\text { Composition of two SG-Bézier swept surfaces by } \\
\text { only } C^{0} \text { continuity conditions. }\end{array}$ & $\begin{array}{l}\text { Two adjacent GBT-Bézier swept surfaces can be } \\
\text { connected by higher } C^{1} \text { and } C^{2} \text { continuity } \\
\text { smoothness. }\end{array}$ \\
\hline 6 & No set of algorithms has been constructed. & $\begin{array}{l}\text { A set of algorithms can be constructed for } \\
\text { explaining how to enforce these engineering } \\
\text { surfaces in practice. }\end{array}$ \\
\hline
\end{tabular}

Anyhow, due to the fixed shape and position relative to the control polygon [1], the traditional Bézier curves and surfaces still have some deficiencies. Practical applications of Bézier curves and surfaces in the area of geometric modeling in engineering are restricted due to these deficiencies. With the help of shape control parameters into Bézier scheme, a remarkable study has been done [2-7] to tackle the problems in adjusting and controlling the shapes of Bézier curves and surfaces. Using a recursive approach, Yan and Liang [8] defined the Bézier curve and rectangular Bézier with single shape control parameters which are based on a new kind of polynomial basis functions. The presence of shape control parameter in [8] enhance the control over the shape and position of the proposed curves and surfaces. Based on the basis functions proposed by Yan and Liang, Hu et al. joined the two Bézier-like curve and surface segments with $G^{2}$ continuity constrains in [9]. Hu et al. [10] presented a new efficient technique for quickly designing generalized Bézier rotation surfaces using shape-adjustable generalized Bézier curves along various shape control parameters. Some advantages over existing scheme [11] are presented in Table 1.

However, the traditional Bézier curves and surfaces have another limitation due to their polynomial representation because the polynomial functions have low smoothness. Thus many scholars have tried to resolve this issue in a non-polynomial function space. Considerable research has been accomplished during the last few years with the help of trigonometric functions or the blending of polynomial and trigonometric functions for the description of curves and surfaces. Trigonometric B-splines were firstly presented by Schoenberg in [12], and the iterative relationship of random order trigonometric B-splines was settled in [13]. The trigonometric polynomials play a key role in the areas of CAGD, medicine, and electronics $[14,15]$. In recent times, trigonometric polynomials have also acquired remarkable attention in the area of geometric modeling in engineering. A new technique for the construction of quartic quasi-Bézier rotation surfaces was presented by Hu in [16] using various shape control parameters. Yan [17] expressed cubic trigonometric nonuniform spline curves and surfaces. Using appropriate shape control parameters, Sharma suggested quartic trigonometric, quasi-quartic trigonometric, and a class of Bézier-type cubic trigonometric curves and surfaces sequentially in [18, 19], and [20].

To solve some problems in the construction of symmetric revolutionary curves and surfaces, a new approach was defined by BiBi et al. [21] using generalized hybrid trigonomet- 
ric Bézier curve (shortly, GHT-Bézier) with shape control parameters. Some free-form complex curves with restriction of parametric continuity were also constructed by using these GHT-Bézier curves. Furthermore, to illustrate the efficiency of the scheme, the authors also presented some composite symmetric curves and surfaces using symmetric formulas and parametric continuity conditions. In 2020, Maqsood et al. defined generalized trigonometric Bézier (shortly, GT-Bézier) curves and surfaces with some shape control parameters and also discussed their continuity constraints and some applications of geometric modeling in engineering [22]. A new approach about $G^{3}$ continuity of GHTBézier curves is proposed by BiBi et al. in [23]. Modeling of free form complex figures and sketching is also presented in this literature by $C^{k}$ and $G^{k}(k \leq 3)$ continuity.

The GBT-Bézier curves based on GBTB functions with two shape parameters will be constructed in this research work. Furthermore, taking these GBT-Bézier curves, six types of engineering surfaces like GBT-Bézier cylinders, GBT-Bézier bilinear surfaces, GBTBézier ruled surfaces, GBT-Bézier swung surfaces, GBT-Bézier swept surfaces, and GBTBézier rotation surfaces will be formulated.

As an alternative technique of representing curves and surfaces, these proposed curves and surfaces not only have the valuable features of Bézier curves and surfaces but also allow an efficient shape modification technique. As an alternative technique of representing curves and surfaces in Bézier approach, these GBT-Bézier curves and surfaces not only have the valuable features of the traditional Bézier curves and surfaces but will also prove an efficient shape modification technique in the area of computer vision, manufacturing industry, computer animation, and multimedia technology.

Some technical contributions are made in this study which are as follows:

- Construction of a tensor product GBT-Bézier surface by a new set of GBTB functions with two shape parameters.

- Construction of some computer-based engineering surfaces using GBT-Bézier with shape parameters.

- The complex computer-based engineering surfaces using GBT-Bézier patches are composed by $C^{k}(k=1,2,3)$ continuity conditions.

- Algorithms of all complex GBT-Bézier engineering surfaces are constructed for easy understanding of interested readers.

This research work is organized as follows: In Sect. 2, basic definitions and properties of GBTB functions and their corresponding GBT-Bézier curves and surfaces are given. On the bases of proposed GBT-Bézier curves along with their shape control parameters, six different engineering surfaces are designed in Sect. 3. Concluding remarks on this research are given in Sect. 4.

\section{Definitions and features of GBT-Bézier curves and surfaces}

\subsection{Generalized blended trigonometric Bernstein-like basis functions}

In this section, the generalized blended trigonometric Bernstein-like basis functions (GBTB) are defined.

Definition 1 For any $\mu, v(-1 \leq \mu, v \leq 1)$ and $z(-1 \leq z \leq 1)$,

$$
\left\{\begin{array}{l}
f_{0,2}(z)=\left(1-\sin \left(\frac{\pi}{2} z\right)\right)\left(1-\mu \sin \left(\frac{\pi}{2} z\right)\right), \\
f_{1,2}(z)=\left(1-f_{0,2}(z)-f_{2,2}(z)\right), \\
f_{2,2}(z)=\left(1-\cos \left(\frac{\pi}{2} z\right)\right)\left(1-v \cos \left(\frac{\pi}{2} z\right)\right),
\end{array}\right.
$$




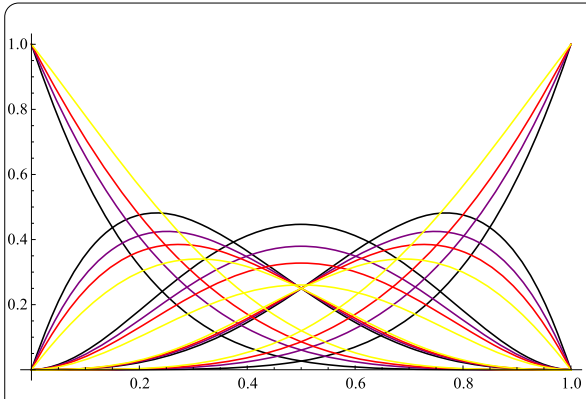

(a) $m=4$

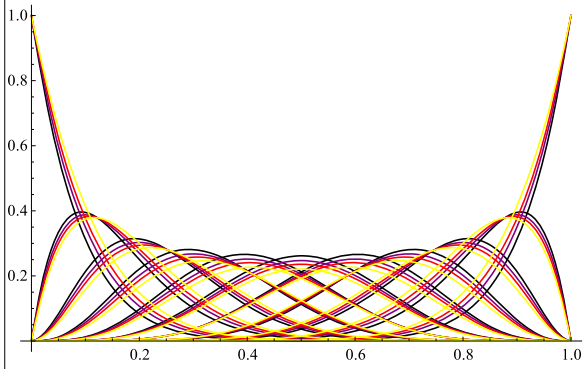

(c) $m=10$

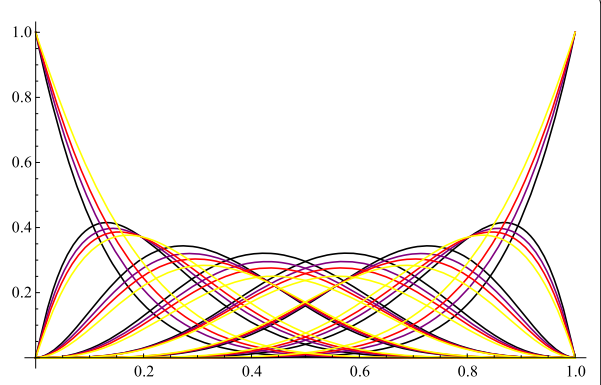

(b) $m=7$

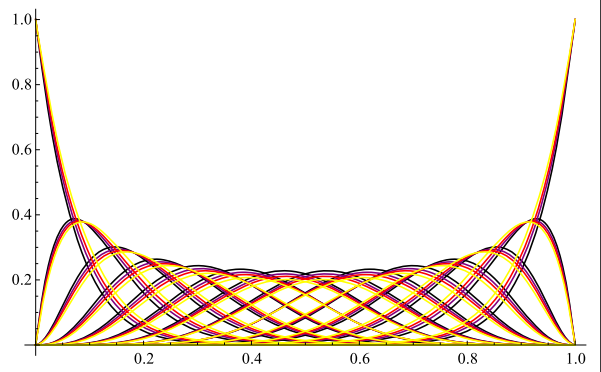

(d) $m=13$

Figure 1 GBTB basis functions of different order with different shape parameters

are known as second order GBTB basis functions with shape parameters $\mu, v$. For any integer $m(m \geq 3)$, the functions $f_{k, m}(z), k=0,1,2, \ldots, m$, defined as

$$
f_{k, m}(z)=(1-z) f_{k, m-1}(z)+z f_{k-1, m-1}(z)
$$

are GBTB basis functions of order $m$ [24]. In particular, when $k=-1$ or $k>n$, the functions $f_{k, m}(z)=0$. Figure 1 depicts the graphs of GBTB basis functions of different order with shape parameters as $\mu, v=0.9$ (black), 0.25 (purple), -0.25 (red), and -0.9 (yellow).

Theorem 1 The GBTB basis functions have the following characteristics:

1. Degeneracy: GBTB basis functions of order $m$ become just like the classical Bernstein basis functions of order $m$ by setting $\mu, v=1$ and $\sin \left(\frac{\pi}{2} z\right), 1-\cos \left(\frac{\pi}{2} z\right)=z$.

2. Nonnegativity: $\forall \mu, v(-1 \leq \mu, v \leq 1)$, the functions $f_{k, m}(z) \geq 0(k=0,1,2, \ldots, m)$.

3. Partition of unity:The sum of all GBTB basis functions of degree $m$ is equal to one.

4. Symmetry: The functions $f_{k, m}(z)(k=0,1,2, \ldots, m)$ are symmetric when $\mu=v$, i.e.,

$$
f_{m-k, m}(z, \mu, v)=f_{k, m}(1-z, \mu, v) \text {. }
$$

5. Derivative at the end points: $f_{k, m}^{\prime}(0), f_{k, m}^{\prime}(1), f_{k, m}^{\prime \prime}(0), f_{k, m}^{\prime \prime}(1), f_{k, m}^{\prime \prime \prime}(0)$, and $f_{k, m}^{\prime \prime \prime}(1)$.

Proof The proofs of all above results are as mentioned in [24]. 


\subsection{Construction of GBT-Bézier curves with shape parameters}

Definition 2 For any control points $Q_{k} \in R^{m}, m=2,3 ; k=0,1, \ldots, m$, the expression

$$
F_{m}(z ; \mu, \nu)=\sum_{k=0}^{m} f_{k, m}(z) Q_{k}, \quad 0 \leq z \leq 1,
$$

is termed as GBT-Bézier curves, where $f_{k, m}(z)$ are GBTB basis functions (2).

The GBT-Bézier curves take over most advantageous features of the traditional Bézier curves, including symmetry, convex hull property, variation diminishing property, geometric invariance, shape adjustable property, and the terminal properties $F(0 ; \mu, \nu)$, $F(1 ; \mu, v), F^{\prime}(0 ; \mu, v), F^{\prime}(1 ; \mu, v), F^{\prime \prime}(0 ; \mu, v), F^{\prime \prime}(1 ; \mu, v), F^{\prime \prime \prime}(0 ; \mu, v)$, and $F^{\prime \prime \prime}(1 ; \mu, v)$. The terminal properties of GBT-Bézier curves indicate that the GBT-Bézier curve segments interpolate to the both end points of their convex hull, and the values of their derivatives at both end points on GBT-Bézier curves will be easily modified by adjusting the shape parameters $\mu, v$ in their respective value range, which brings a significant ease to smooth joining [24].

Theorem 2 The necessary and sufficient $C^{3}$ continuity conditions among two GBT-Bézier curve segments $F(z ; \mu, v)=\sum_{k=0}^{m} Q_{k} f_{k, m}(z)$ and $F_{1}\left(z ; \mu_{1}, v_{1}\right)=\sum_{k=0}^{n} Q 1_{k} f_{k, n}(z)$ with control points $Q_{0}, Q_{1}, Q_{2}, \ldots, Q_{m}, m \geq 3$, and $Q 1_{0}, Q 1_{1}, Q 1_{2}, \ldots, Q 1_{n}, n \geq 3$, respectively are described as follows:

1. $Q_{m}=Q 1_{0}$ for $C^{0}$ continuity.

2. For $C^{1}$ continuity,

$$
\left\{\begin{array}{l}
Q 1_{0}=Q_{m} \\
Q 1_{1}=Q_{m}+\frac{(2(m-2)+\pi(1+v))}{2(n-2)+\pi\left(1+\mu_{1}\right)}\left(Q_{m}-Q_{m-1}\right) .
\end{array}\right.
$$

3. For $C^{2}$ continuity,

$$
\left\{\begin{aligned}
Q 1_{0}= & Q_{m}, \\
Q 1_{1}= & Q_{m}+\frac{(2(m-2)+\pi(1+v))}{2(n-2)+\pi\left(1+\mu_{1}\right)}\left(Q_{m}-Q_{m-1}\right), \\
Q 1_{2}= & Q_{m}+\frac{1}{a_{1}}\left[a_{2}\left(Q_{m}-2 Q_{m-1}+Q_{m-2}\right)-\pi^{2}(1-\mu)\left(Q_{m-1}-Q_{m-2}\right)\right. \\
& \left.+a_{3}\left(Q_{m}-Q_{m-1}\right)\right] .
\end{aligned}\right.
$$

4. For $C^{3}$ continuity,

$$
\left\{\begin{aligned}
Q 1_{0}= & Q_{m} \\
Q 1_{1}= & Q_{m}+a\left(Q_{m}-Q_{m-1}\right), \\
Q 1_{2}= & Q_{m}+\frac{1}{a_{1}}\left[a_{2}\left(Q_{m}-2 Q_{m-1}+Q_{m-2}\right)-\pi^{2}(1-\mu)\left(Q_{m-1}-Q_{m-2}\right)\right. \\
& \left.+a_{3}\left(Q_{m}-Q_{m-1}\right)\right], \\
Q 1_{3}= & Q_{m}+\frac{1}{b_{1}}\left[b_{2}\left(Q_{m}-3 Q_{m-1}+3 Q_{m-2}-Q_{m-3}\right)+\frac{a_{2}}{a_{1}} b_{3}\left(Q_{m}-2 Q_{m-1}+Q_{m-2}\right)\right. \\
& \left.-\frac{\pi^{2}\left(1-\mu_{1}\right) b_{3}}{a_{1}}\left(Q_{m-1}-Q_{m-2}\right)-b_{4}\left(Q_{m}-Q_{m-1}\right)\right],
\end{aligned}\right.
$$


where

$$
\begin{aligned}
& a=\frac{2(m-2)+\pi(1+v)}{2(n-2)+\pi\left(1+\mu_{1}\right)} \\
& a_{1}=4(n-2)\left(n-3+\pi\left(1+\mu_{1}\right)\right)+\pi^{2}\left(1-v_{1}\right), a_{2}=4(m-2)(m-3+\pi(1+v)), \\
& a_{3}=\left(2 a_{1}+\pi^{2}\left(1+2 \mu_{1}-v_{1}\right)+2 \pi^{2} v\right), \\
& b_{1}=4(n-2)(n-3)\left(2(n-4)+3 \pi\left(1+\mu_{1}\right)\right)+6(n-2) \pi^{2}\left(1-v_{1}\right), \\
& b_{2}=4(m-2)(m-3)(2(m-4)+3 \pi(1+v)), \\
& b_{3}=\left(3 c_{1}+12(n-2) \pi^{2}\left(1-\mu_{1}-v_{1}\right)\right), \\
& b_{4}=\frac{a_{3} b_{3}}{a_{1}}-a\left(c_{1}+12(n-2) \pi^{2}\left(1-\mu_{1}-4 v_{1}\right)+\pi^{3}\left(1+\mu_{1}\right)\right)+\pi^{3}(1+v), \\
& c_{1}=4(n-2)(n-3)\left(2(n-4)+3 \pi\left(1+\mu_{1}\right)\right) .
\end{aligned}
$$

Proof The proofs of all the above results are as described in [24].

\subsection{Formation of GBT-Bézier surfaces with shape parameters}

Definition 3 The tensor product GBT-Bézier surfaces of degree $(m, n)$ with shape parameters $\mu, v, \mu_{1}, v_{1}$ and control points $Q_{k, l} \in R^{3}, k=0,1,2, \ldots, m, l=0,1,2, \ldots, n$, are defined as follows:

$$
T_{m, n}\left(z, z_{1}, \mu, v, \mu_{1}, v_{1}\right)=\sum_{k=0}^{m} \sum_{l=0}^{n} Q_{k, l} f_{k, m}(z) f_{l, n}\left(z_{1}\right), \quad z, z_{1} \in[0,1]
$$

where $f_{k, m}(z), f_{l, n}\left(z_{1}\right)$ are GBTB basis functions of order $m$ and $n$ respectively with shape parameters $\mu, v, \mu_{1}, v_{1}$. As the GBT-Bézier surfaces are defined on the bases of GBTBézier curves, so they have all the features that the GBT-Bézier curves have.

The tensor product GBT-Bézier surfaces shares all the properties with classical tensor product Bézier surfaces except for the shape adjustable property which is superior to the properties of classical tensor product Bézier surfaces.

Figures 2 and 3 represent a GBT-Bézier vase diagram and a bi-cubic GBT-Bézier surface with different values of shape parameters.

\section{Formation of adjustable engineering surfaces}

\subsection{GBT-Bézier cylinder with shape parameters}

Let $F_{m}(z, \mu, v)=\sum_{k=0}^{m} Q_{k} f_{k, m}(z), z \in[0,1]$ be a GBT-Bézier curve of degree $m$ and $\mathbf{V}$ be a $3 D$ unit vector, the representation of the GBT-Bézier cylinder $T\left(z, z_{1}, \mu, v\right)$ is obtained by sweeping $F_{m}(z, \mu, v)$ a distance $\alpha(\alpha>0)$ along $\mathbf{V}$ [25]. For sweep direction representing the parameter by $z_{1}$, clearly the GBT-Bézier cylinder should meet the following requirements:

(i) The curve $T\left(\bar{z}, z_{1}, \mu, v\right)$ is a straight line segment from point $F_{m}(\bar{z}, \mu, v)$ to $F_{m}(\bar{z}, \mu, \nu)+\alpha \mathbf{V}$ for fixed $\bar{z}(0 \leq \bar{z} \leq 1)$. 


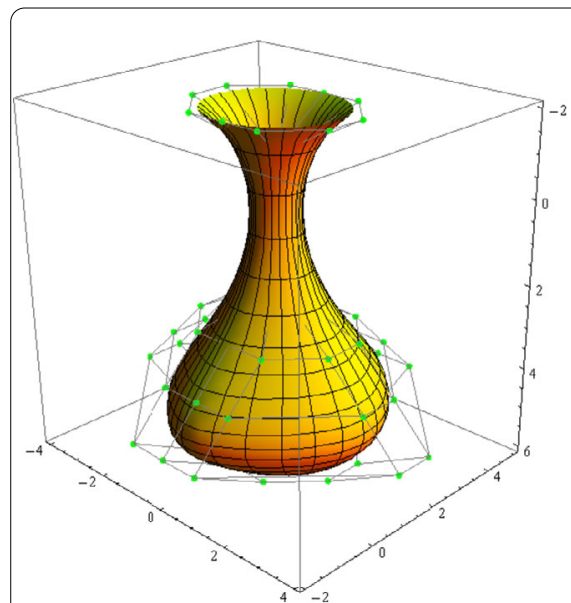

(a) $\forall \mu, \nu=1$

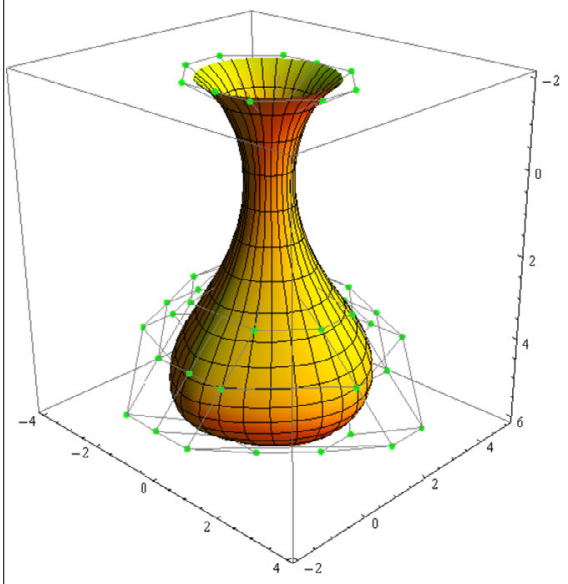

(c) $\forall \mu=1, \nu=-1$

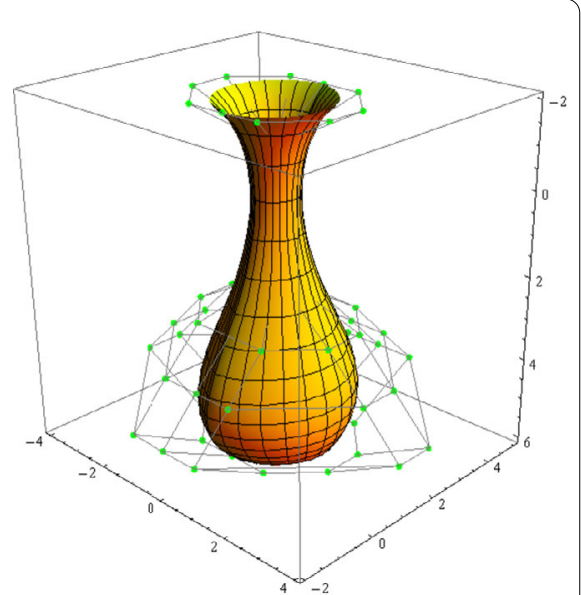

(b) $\forall \mu, \nu=-1$

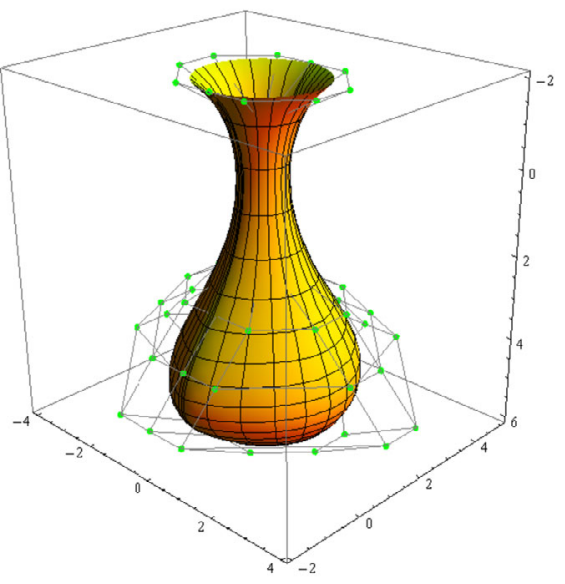

(d) $\forall \mu=-1, \nu=1$

Figure 2 Effect of shape parameters on a GBT-Bézier surface

(ii) For fixed $\bar{z}_{1}\left(0 \leq \bar{z}_{1} \leq 1\right)$, we have

$$
\begin{aligned}
T\left(z, \overline{z_{1}}, \mu, v\right) & =F_{m}(z, \mu, v)+\bar{z}_{1} \alpha \mathbf{V} \\
& =\sum_{k=0}^{m}\left(Q_{k}+\bar{z}_{1} \alpha \mathbf{V}\right) f_{k, m}(z) .
\end{aligned}
$$

Proposition 3.1 The translational invariance property is used to define GBT-Bézier curves in (8). Thus, in the perspective of the aforementioned conditions, the expression of the GBTBézier cylinder can be described as follows:

$$
T_{\text {cylinder }}\left(z, z_{1}, \mu, v\right)=\sum_{k=0}^{m}\left(Q_{k}+z_{1} \alpha \mathbf{V}\right) f_{k, m}(z), \quad z, z_{1} \in[0,1]
$$

where $\alpha>0$ is a certain real constant. Taking $F_{m}(z, \mu, v)$ as directrix of the GBT-Bézier cylinder, expression (9) is called the GBT-Bézier cylinder. 


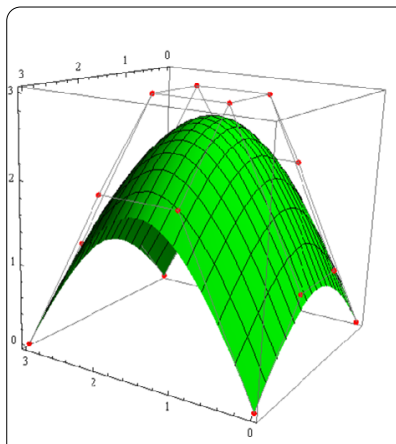

(a) $\mu, \nu, \mu_{1}, \nu_{1}=1$

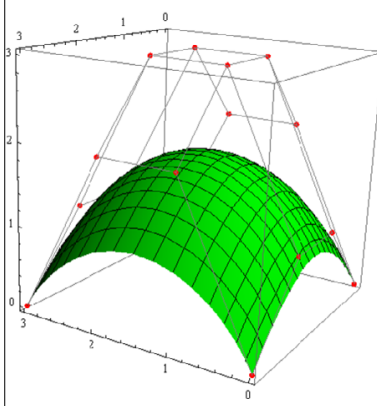

(d) $\mu, \nu=-1, \mu_{1}, \nu_{1}=0.7$

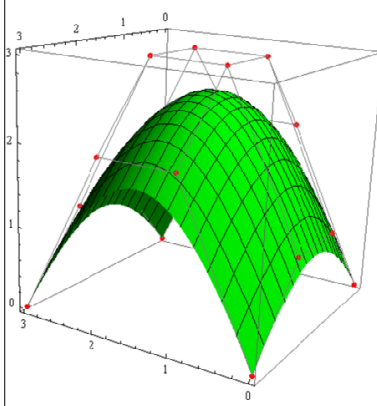

(g) $\mu, \mu_{1}=0.5, \nu, \nu_{1}=1$

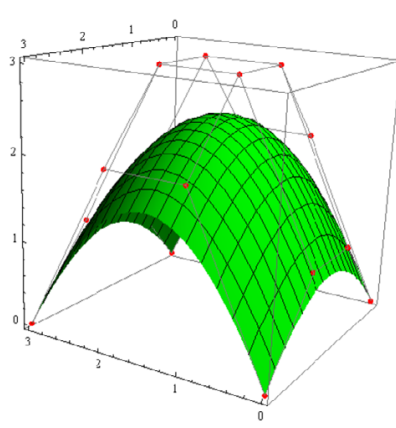

(b) $\mu, \nu, \mu_{1}, \nu_{1}=0.5$

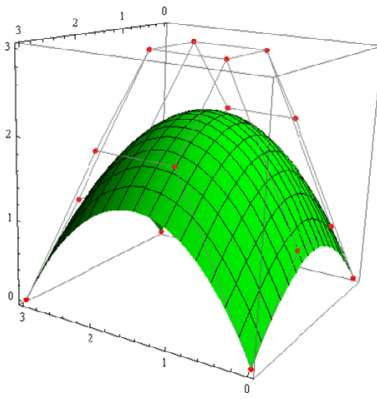

(e) $\mu, \nu=0, \mu_{1}, \nu_{1}=0.7$

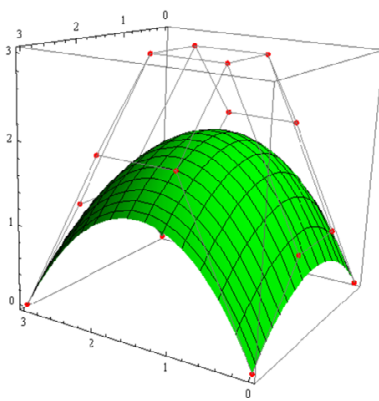

(h) $\mu, \mu_{1}=0.5, \nu, \nu_{1}=-1$

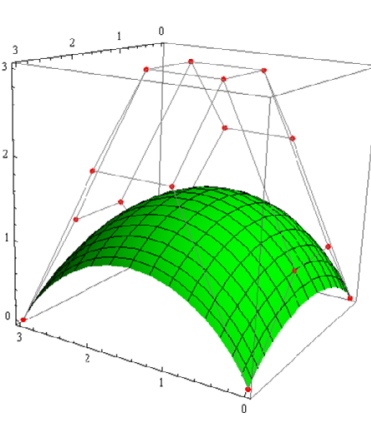

(c) $\mu, \nu, \mu_{1}, \nu_{1}=-1$

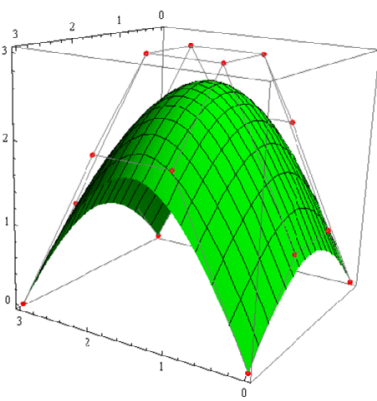

(f) $\mu, \nu=1, \mu_{1}, \nu_{1}=0.7$

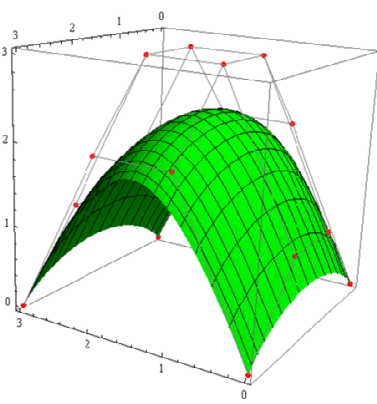

(i) $\mu, \nu=1, \mu_{1}, \nu_{1}=-1$

Figure 3 Effect of shape parameters on bi-cubic GBT-Bézier surfaces

Moreover, the GBT-Bézier cylinder $T_{\text {cylinder }}$ is a surface which is generated by translating a small portion of the straight line from $F_{m}(0)$ to $F_{m}(0)+\alpha \mathbf{V}$ along an assigned curve $F_{m}(z, \mu, v)$. Anyhow, the cylinder in (9) leads to some complications in designing GBTBézier curves and surfaces as it is not a tensor product GBT-Bézier surface. To overcome this problem, equation (9) is transformed into the tensor product GBT-Bézier surface described in (7). It is observed that the $m$ th-degree GBTB basis functions defined by (2) are only described for parameter $m(m \geq 2)$, but they are not given for $m=1$. Thus, extending the definition of GBTB basis functions, we describe the first-degree GBTB basis functions as

$$
f_{k, 1}(z)=(1-z)^{1-k} z^{k} \quad(k=0,1) .
$$


Expression (10) shows that the GBTB basis functions of order one are the traditional Bernstein basis functions of order one.

Theorem 3 A GBT-Bézier cylinder in (9) can also be written in its tensor product form as follows:

$$
T_{\text {cylinder }}\left(z, z_{1}, \mu, v, \mu_{1}, v_{1}\right)=\sum_{k=0}^{m} \sum_{l=0}^{1} Q_{k, l} f_{k, m}(z) f_{l, n}\left(z_{1}\right), \quad z, z_{1} \in[0,1]
$$

where $Q_{k, 0}=Q_{k}, Q_{k, 1}=Q_{k}+d \mathbf{V}, k=0,1, \ldots, m$, are control points, $f_{k, m}(z), f_{l, 1}\left(z_{1}\right), l=0,1$, are GBTB basis functions described by (2) and (10), respectively, and $\mu$, $v$ are shape control parameters.

Proof By taking control points $Q_{k, 0}=Q_{k}$ and $Q_{k, 1}=Q_{k}+\alpha \mathbf{V}=Q_{k, 0}+\alpha \mathbf{V}$ and basis function given in (10), we have

$$
\begin{aligned}
T_{\text {cylinder }}\left(z, z_{1}, \mu, v\right) & =\sum_{k=0}^{m}\left(Q_{k}+z_{1} d \mathbf{V}\right) f_{k, m}(z) \\
& =\sum_{k=0}^{m}\left[Q_{k, 0}+z_{1}\left(Q_{k, 1}-Q_{k, 0}\right)\right] f_{k, m}(z) \\
& =\sum_{k=0}^{m}\left[\left(1-z_{1}\right) Q_{k, 0}+z_{1} Q_{k, 1}\right] f_{k, m}(z) \\
& =\sum_{k=0}^{m} \sum_{l=0}^{1} Q_{k, 1} f_{k, m}(z) f_{l, 1}\left(z_{1}\right) .
\end{aligned}
$$

Therefore, GBT-Bézier cylinder in (9) can be exactly expressed in terms of a tensor product GBT-Bézier surface of degree $(m, 1)$. Hence the proof of the result.

Since a GBT-Bézier curve is the directrix of a GBT-Bézier cylinder, it carries some remarkable benefits of the general Bézier cylinder. Furthermore, the GBT-Bézier cylinder (11) has an edge that by keeping the control mesh unchanged, the GBT-Bézier cylinder can be transformed into any shape merely by changing the shape parameters.

Here, some detailed steps are described to design a GBT-Bézier cylinder.

\section{Algorithm 1}

1. Choose an arbitrary $3 D$ unit vector $\mathbf{V}$ and a GBT-Bézier curve as the directrix of GBT-Bézier cylinder.

2. Describe a GBT-Bézier cylinder in the manner as given in equation (11).

3. Take control points $Q_{k, l}$ as $Q_{k, 0}=Q_{k}, Q_{k, 1}=Q_{k}+\alpha \mathbf{V}(k=0,1,2, \ldots, m)$, where $\alpha$ is the distance along a normal vector of the plane.

4. Select the distance $\alpha$ and draw the GBT-Bézier cylinder with different shape parameters via Wolfram Mathematica software.

5. GBT-Bézier cylinder can also be drawn with varying values of $\alpha$ and keeping the values of shape parameters unchanged. 


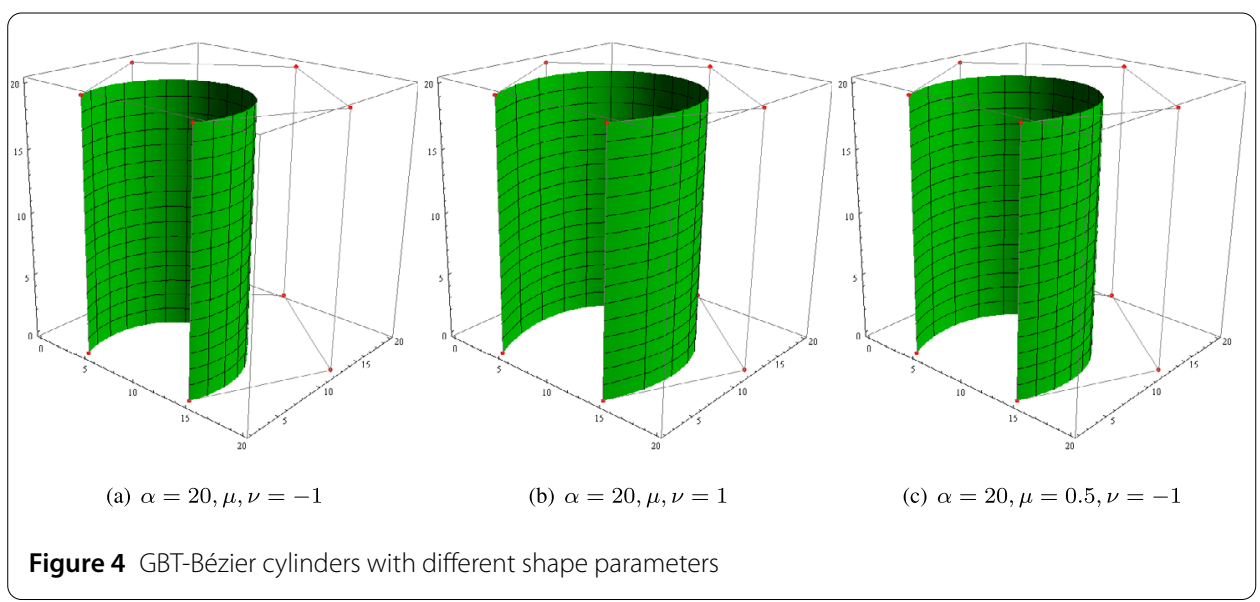

Example 3.1 A model of GBT-Bézier cylinder with GBT-Bézier curve as directrix is given in this example. Suppose that a GBT-Bézier curve $F_{4}(z ; \mu, v)$ is constructed by five control points $Q_{0}=(5,2,0), Q_{1}=(0,12,0), Q_{2}=(10,20,0), Q_{3}=(20,12,0), Q_{4}=(15,2,0)$. Now we generate a GBT-Bézier cylinder by interpreting $F_{4}(z ; \mu, \nu)$ a distance $\alpha$ with the normal vector of the plane (here $\alpha=20, \mathbf{V}=(0,0,1))$ and taking the curve $F_{4}(z ; \mu, v)$ as the directrix. By Theorem 3, the equation of the GBT-Bézier cylinder can be represented as follows:

$$
T_{\text {cylinder }}\left(z, z_{1}, \mu, v\right)=\sum_{k=0}^{4} \sum_{l=0}^{1} Q_{k, l} f_{k, 4}(z) f_{l, 1}\left(z_{1}\right), \quad z, z_{1} \in[0,1]
$$

where $Q_{k, 0}=Q_{k}, Q_{k, 1}=Q_{k}+(0,0,20)(k=0,1, \ldots, 4)$ are control net points. Figure 4 represents the GBT-Bézier cylinders with 10 mesh points for fixed values of $\alpha=20$ and different shape parameters $\mu, v$ which clearly make its confined convex hull.

A closed GBT-Bézier cylinder is illustrated in Fig. 5 to show the impact of $\alpha$ and shape control parameters $\mu, v$ on GBT-Bézier cylinder, whereas the $C^{2}$ continuity of two GBTBézier cylinders is illustrated in Fig. 6 with shape control parameters $\mu, \mu_{1}, v, v_{1}$. Figures 4, 5 , and 6 present that the effect of shape parameters on GBT-Bézier cylinders (open or close) is the same as the effect on GBT-Bézier curves and surfaces.

\subsection{GBT-Bézier bi-linear surface with shape parameters}

The authors in [25] proposed a nonuniform rational B-spline (NURBS) representation of the surface (nonrational) called bi-linear surface by bi-linearly interpolating among four line segments, $Q_{0,0} Q_{1,0}, Q_{0,1} Q_{1,1}, Q_{0,0} Q_{0,1}$, and $Q_{1,0} Q_{1,1}$ using four control points $Q_{0,0}, Q_{1,0}$, $Q_{0,1}, Q_{1,1}$ in a $3 D$ space which is given by

$$
T_{\text {bi-linear }}\left(z, z_{1}, \mu, v, \mu_{1}, v_{1}\right)=\sum_{k=0}^{1} \sum_{l=0}^{1} Q_{k, l} N_{k, 1}(z) N_{l, 1}\left(z_{1}\right), \quad z, z_{1} \in[0,1]
$$

where $\mathbf{Z}=\mathbf{Z}_{\mathbf{1}}=\{0,0,1,1\}$ is the node vector and $Q_{k, l}, k, l \in 0,1$ are taken as control points. Among opposite boundary lines, the bi-linear surface (14) performs a clear linear interpolation in both direction [25]. 


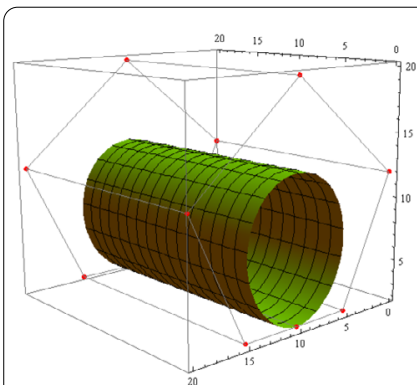

(a) $\alpha=20, \mu, \nu=-1$

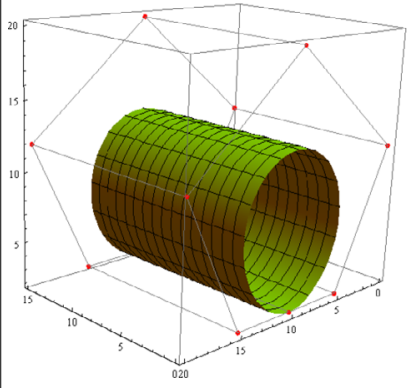

(d) $\alpha=15, \mu, \nu=1$

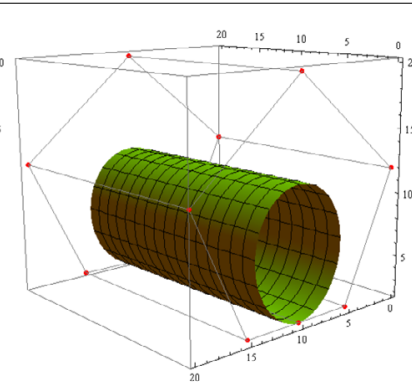

(b) $\alpha=20, \mu=0.1, \nu=0$

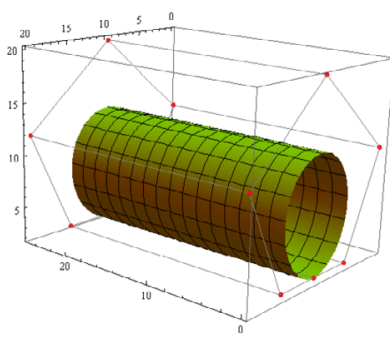

(e) $\alpha=25, \mu, \nu=1$

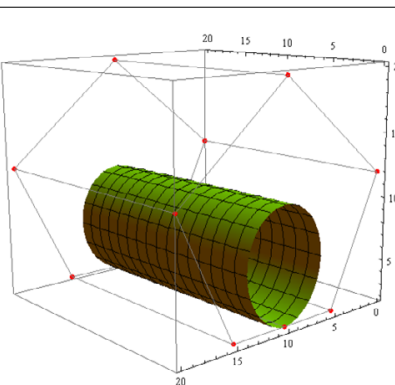

(c) $\alpha=20, \mu=1, \nu=0.9$

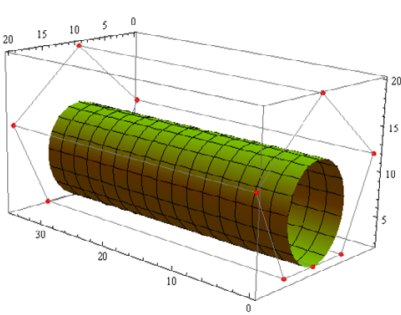

(f) $\alpha=35, \mu, \nu=1$

Figure 5 GBT-Bézier cylinders with different values of $\mu, v$, and $\alpha$

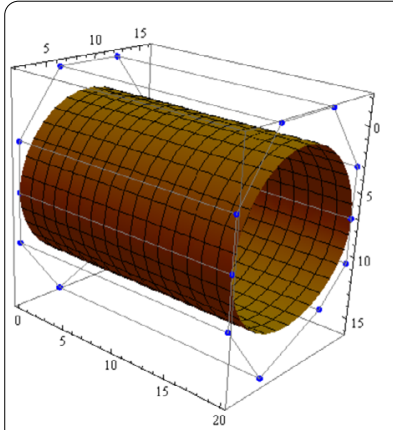

(a) $\mu, \mu_{1}=0.5, \nu, \nu_{1}=0.1$

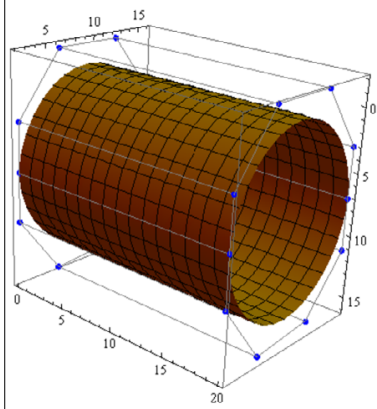

(d) $\mu, \nu=0.9, \mu_{1}, \nu_{1}=0.8$

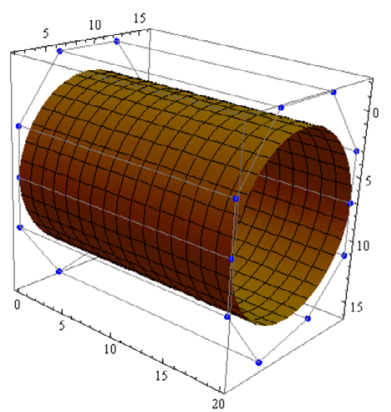

(b) $\mu \mu_{1}=0.5, \nu, \nu_{1}=0.6$

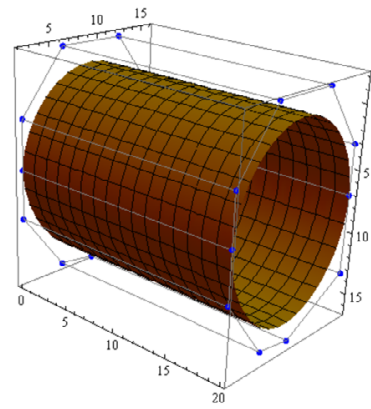

(e) $\mu, \nu=0.9, \mu_{1}, \nu_{1}=-0.5$

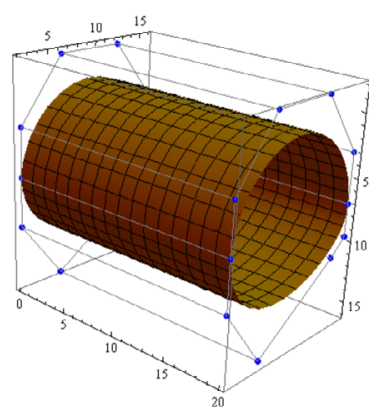

(c) $\mu, \mu 1=0.5, \nu, \nu_{1}=-0.7$

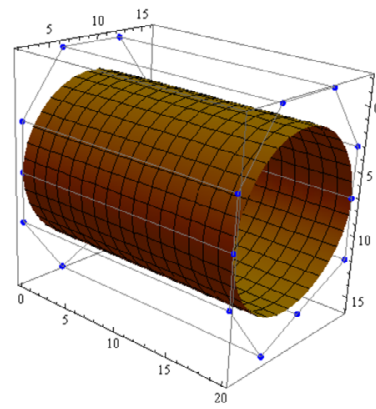

(f) $\mu, \mu_{1}=-0.2, \nu, \nu_{1}=0.4$

Figure $6 C^{2}$ continuity between two GBT-Bézier cylinders 
Proposition 3.2 The characteristics of the bi-linear surface (14) are as follows: (a) given angular points are interpolated by the surface; (b) line segments are taken as boundary curves; $(c)$ the curves $T\left(\bar{z}, z_{1}\right)$ or $T\left(z, \bar{z}_{1}\right)$ are the line segments for fixed $\bar{z}$ or $\bar{z}_{1}$. The transformation of a bi-linear surface in terms of tensor product GBT-Bézier surface is given by

$$
T_{\text {bi-linear }}\left(z, z_{1}, \mu, v, \mu_{1}, v_{1}\right)=\sum_{k=0}^{1} \sum_{l=0}^{1} Q_{k, l} f_{k, 1}(z) f_{l, 1}\left(z_{1}\right), \quad z, z_{1} \in[0,1],
$$

where $f_{k, 1}(z), f_{l, 1}\left(z_{1}\right)(k, l=0,1)$ are basis functions defined in (10).

However, the bi-linear surfaces described in (14) and (15) cannot be modified. This inconvenience of shape adjustment is due to the given four angular points. So, we need to generalize the classical bi-linear surface (14) into a GBT-Bézier bi-linear surface which is defined by taking a GBT-Bézier curve. Some properties of such surfaces are given as follows:

1. Angular points are interpolated by the surface;

2. Line segments are taken as the boundary curves of the surface;

3. $T_{m, n}\left(\bar{z}, z_{1}\right)$ or $T_{m, n}\left(z, \bar{z}_{1}\right)$ is a GBT-Bézier curve for fixed $\bar{z}$ or $\bar{z}_{1}$;

4. The model of GBT-Bézier surface is used by the bi-linear surface.

From the characteristics of GBT-Bézier curves and surfaces, the actual procedure for developing a GBT-Bézier bi-linear surface is described. The steps of the algorithm are as follows.

\section{Algorithm 2}

1. In a $3 D$ space, take $Q_{0,0}, Q_{m, 0}, Q_{0, n}, Q_{m, n}$ to be the four control points. These points are used as the angular points of the GBT-Bézier surface.

2. Split the line segments $Q_{0,0} Q_{m, 0}, Q_{0, n} Q_{m, n}$ and $Q_{0,0} Q_{0, n}, Q_{m, 0} Q_{m, n}$ into $m$ and $n$ same parts (an irregular division can also be made) accordingly and indicate the central partition points as $Q_{1,0}, Q_{2,0}, \ldots, Q_{m-1,0}, Q_{1, n}, Q_{2, n}, \ldots, Q_{m-1, n}$ and $Q_{0,1}, Q_{0,2}, \ldots, Q_{0, n-1}, Q_{m, 1}, Q_{m, 2}, \ldots, Q_{m, n-1}$. Moreover all the central partition points and $Q_{0,0}, Q_{m, 0}, Q_{0, n}, Q_{m, n}$ defined above are used as the exterior control mesh points of the GBT-Bézier surface.

3. Connect the control points achieved in step 1 and 2 with the remaining mesh points $Q_{k, l}(k=1,2, \ldots, m-1 ; l=1,2, \ldots, n-1)$ to form the following GBT-Bézier bi-linear:

$$
T_{\text {bi-linear }}\left(z, z_{1}, \mu, \nu, \mu_{1}, v_{1}\right)=\sum_{k=0}^{m} \sum_{l=0}^{n} Q_{k, l} f_{k, m}(z) f_{l, n}\left(z_{1}\right), \quad z, z_{1} \in[0,1] .
$$

Modeling example of a third degree GBT-Bézier bi-linear surface is given below.

Example 3.2 Let $Q_{0,0}=(0,0,0), Q_{3,0}=(3,0,0), Q_{0,3}=(0,3,0), Q_{3,3}=(3,3,0)$ be the four angular points of the bi-linear surface and

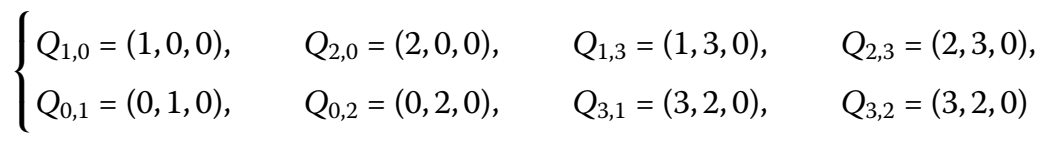




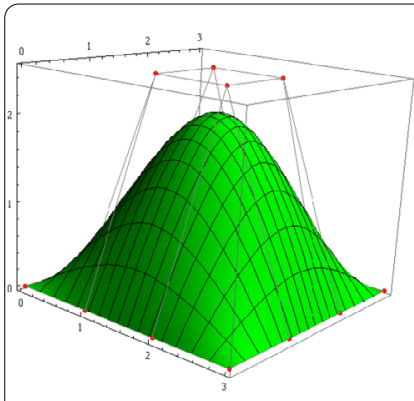

(a) $\mu, \nu, \mu_{1}, \nu_{1}=1$

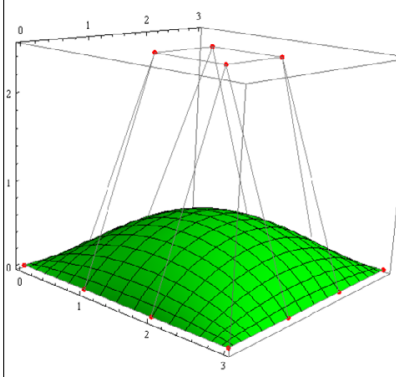

(d) $\mu, \nu, \mu_{1}, \nu_{1}=-1$

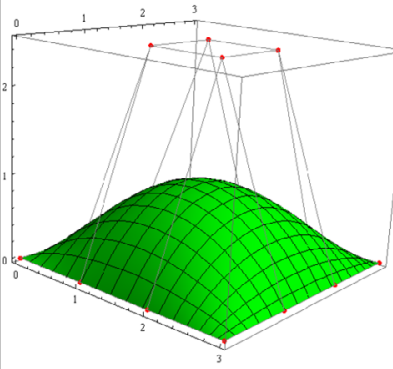

(g) $\mu, \mu_{1}=0, \nu, \nu_{1}=-1$

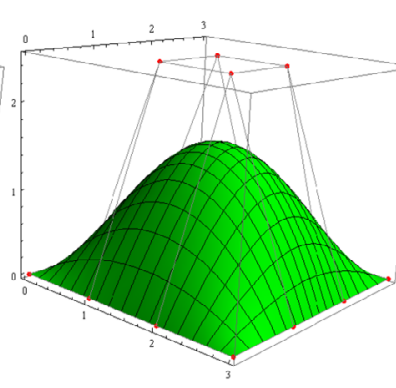

(b) $\mu, \nu=0, \mu_{1}, \nu_{1}=1$

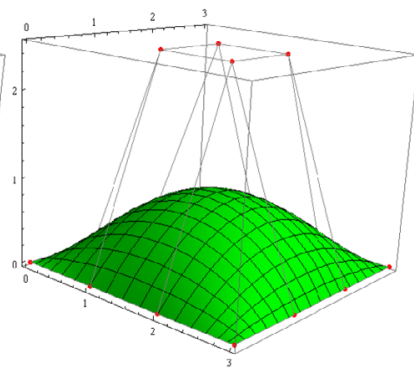

(e) $\mu, \nu=-1, \mu_{1}, \nu_{1}=0$

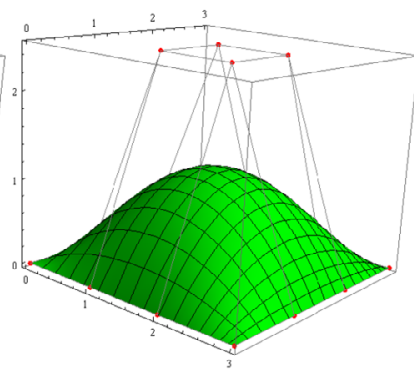

(h) $\mu, \mu_{1}=0.5, \nu, \nu_{1}=-1$

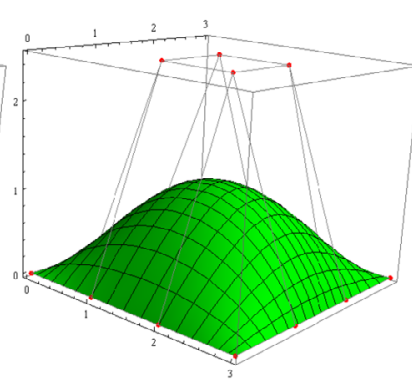

(c) $\mu, \nu=-1, \mu_{1}, \nu_{1}=1$

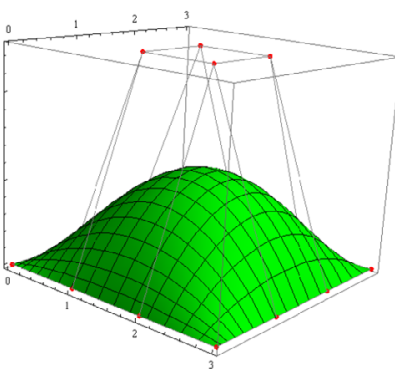

(f) $\mu, \nu=-1, \mu_{1}, \nu_{1}=0.5$

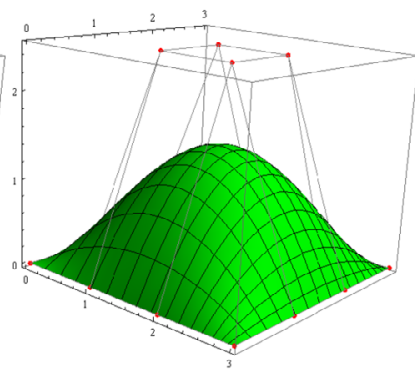

(i) $\mu, \nu=1, \mu_{1}, \nu_{1}=-1$

Figure 7 GBT-Bézier bi-linear surfaces with different shape parameters

be the eight central partition points produced by dividing the line segments $Q_{0,0} Q_{3,0}$, $Q_{0,3} Q_{3,3}, Q_{0,0} Q_{0,3}$, and $Q_{3,0} Q_{3,3}$ into three equal parts. Decisively, the remaining control points $Q_{1,1}=(1,1,3), Q_{2,1}=(2,1,3), Q_{1,2}=(1,2,3), Q_{2,2}=(2,2,3)$ can be chosen freely.

By using control points and equation (15), the GBT-Bézier bi-linear surface can be designed as shown in Fig. 7 and Fig. 8. Bi-linear surfaces have sixteen control points, in which the exterior twelve control mesh points are coplanar. Furthermore, Fig. 8 exhibits the graphs of the GBT-Bézier bi-linear surfaces that have four non-coplanar boundary curves.

\subsection{GBT-Bézier ruled surface along shape parameters}

Let $b_{k}(z)(k=1,2)$ be any two space curves (B-spline curves, Bézier curves, or NURBS curves), then in $z_{1}$ ruled direction, a ruled surface $T\left(z, z_{1}\right)$ [25] is created if a linear interpolation is implemented among iso-parametric points of the two curves $b_{1}(z)$ and $b_{2}(z)$. Also, a ruled surface has an astonishing property that, for fixed $z, T\left(\bar{z}, z_{1}\right)$ is a line segment joining points $b_{1}(\bar{z})$ and $b_{2}(\bar{z})$ (line segments are called straight generatrices, whereas the 


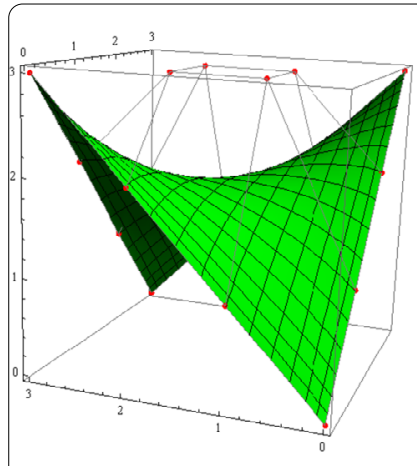

(a) $\mu, \nu, \mu_{1}, \nu_{1}=-1$

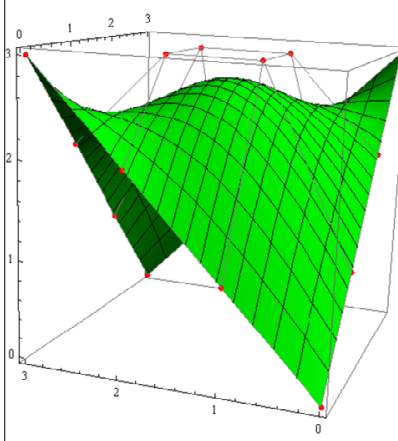

(d) $\mu, \nu, \mu_{1}, \nu_{1}=1$

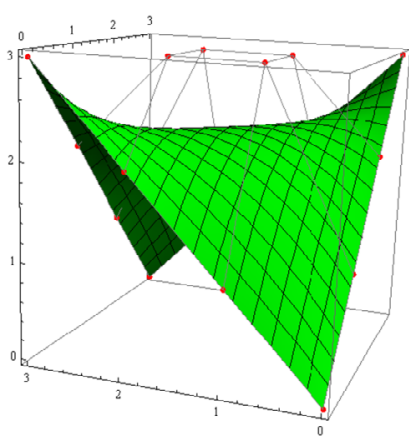

(b) $\mu, \nu, \mu_{1}, \nu_{1}=0$

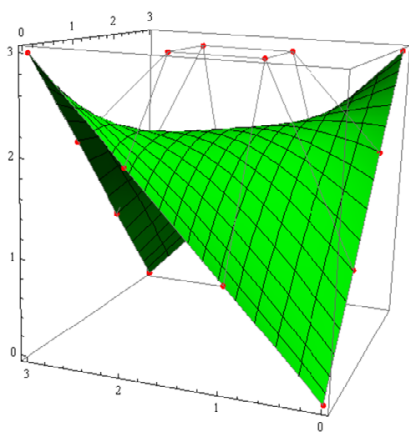

(e) $\mu, \nu=-1, \mu_{1}, \nu_{1}=1$

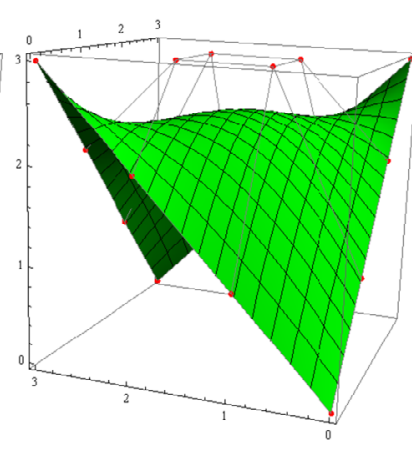

(c) $\mu, \nu, \mu_{1}, \nu_{1}=0.5$

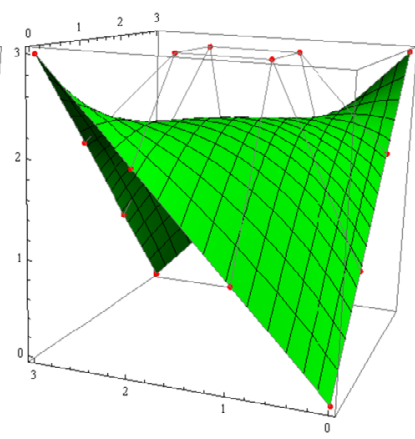

(f) $\mu, \nu=1, \mu_{1}, \nu_{1}=-0.5$

Figure 8 GBT-Bézier bi-linear surfaces with non-planar straight line boundaries

curves $b_{1}(z)$ and $b_{2}(z)$ are named as guide lines, for short GLs). This portion particularly demonstrates how the ruled surface with shape parameters is expressed using tensor product GBT-Bézier surface design. The necessary condition to express the ruled surface into tensor product form is that both should have the same degree. Let

$$
\left\{\begin{array}{l}
F_{m, 0}(z, \mu, v)=\sum_{k=0}^{m} f_{k, m}^{0}(z) Q_{0, k}, \quad 0 \leq z \leq 1, \\
F_{m, 1}\left(z, \mu_{1}, v_{1}\right)=\sum_{k=0}^{m} f_{k, m}^{1}(z) Q_{1, k}, \quad 0 \leq z \leq 1,
\end{array}\right.
$$

be the two GLs of the ruled surface of degree $m$, where $f_{k, m}^{l}(z), k=0,1, \ldots, m, l=0,1$, are the GBTB functions described by (2). The GBT-Bézier ruled surfaces $T_{\text {ruled }}\left(z, z_{1}\right)$ should fulfil the following two conditions according to its definition [25]:

(1) For fixed value of $\bar{z}$, the curve $T_{\text {ruled }}\left(\bar{z}, z_{1}\right)$ is a line segment.

(2) The guild lines fulfil the $T_{\text {ruled }}(z, 0)=F_{m, 0}(z, \mu, v)$ and $T_{\text {ruled }}(z, 1)=F_{m, 1}\left(z, \mu_{1}, v_{1}\right)$.

In accordance with the definition of GBT-Bézier surfaces, the equation of the GBT-Bézier ruled surfaces $T_{\text {ruled }}\left(z, z_{1}\right)$ that meet the aforementioned requirement is

$$
T_{\text {ruled }}\left(z, z_{1}, \mu, \nu, \mu_{1}, v_{1}\right)=\sum_{l=0}^{1}\left(f_{l, 1}\left(z_{1}\right) \sum_{k=0}^{m} f_{k, m}(z) Q_{l, k}\right), \quad 0 \leq z, z_{1} \leq 1,
$$

where $\mu, \mu_{l}, v, v_{l}$ are the shape parameters; $Q_{l, k}(l=0,1 ; k=0,1, \ldots, m)$ are the mesh points defined by (17) and $f_{k, m}(z)$ and $f_{l, 1}\left(z_{1}\right)$ are the GBTB basis functions given in (2) and (10), respectively. Equation (18) represents the tensor product GBT-Bézier surface in the form 
of a GBT-Bézier modified ruled surface. The GBT-Bézier ruled surfaces defined in (18) take over all characteristics and benefits of GBT-Bézier surfaces because these surfaces are expressed in the form of tensor product GBT-Bézier surfaces and the GLs are two GBTBézier curves that have the same degree. The NURBS modeled ruled surface defined in [25] has some limitations, such like complex mathematics, ambiguous weight elements, the annoyance of finding the surface order and the node vector [26]. On the other hand, the required surface has some benefits including straightforward computation, obvious implication of the shape parameters, and the free choice of vertexes.

Proposition 3.3 It is worth recalling that the straight generatrices of a GBT-Bézier ruled surfaces are the straight lines that are obtained by joining iso-parametric points, instead of joining the same length of an arc of the two GLs. Generally, joining the same length of an arc of any two GLs gives us a different ruled surface.

Proposition 3.4 The technique of splicing GBT-Bézier surfaces can be utilized to generate all types of complicated combined GBT-Bézier ruled surfaces for the reason that the GBTBézier ruled surface is a special case of tensor product GBT-Bézier surface. In this technique, the two adjacent GBT-Bézier ruled surfaces satisfy $C^{1}, C^{2}$, and $C^{3}$ continuity conditions because both GLs of the ruled surfaces satisfy these conditions (see Fig. 10 and Fig. 11).

The algorithm for designing a GBT-Bézier ruled surface is as follows.

\section{Algorithm 3}

1. Consider any two GLs in the form of GBT-Bézier curves.

2. Use tensor product form of the GBT-Bézier ruled surface as given in (18).

3. The guild lines must satisfy the conditions $T_{\text {ruled }}(z, 0)=F_{m, 0}(z, \mu, v)$ and $T_{\text {ruled }}(z, 1)=F_{m, 1}\left(z, \mu_{1}, v_{1}\right)$.

4. Take the control points $Q_{i, j}$ in a $3 D$ plane according to designer choice.

5. Draw the GBT-Bézier ruled surface in Wolfram Mathematica software with suitable values of shape parameters.

6. Two adjacent GBT-Bézier ruled surfaces can be connected by $C^{1}, C^{2}$, and $C^{3}$ continuity conditions given in Theorem 2.

Example 3.3 Let $F_{4, l}\left(z, \mu_{1}, v_{1}\right)(l=0,1)$ be two GBT-Bézier curves of degree 4 in a 3dimensional space, then from equation (18) the equation of the GBT-Bézier ruled surfaces formed by these GBT-Bézier curves as the GLs is given by

$$
T_{\text {ruled }}\left(z, z_{1}, \mu_{0}, \nu_{0}, \mu_{1}, \nu_{1}\right)=\sum_{l=0}^{1}\left(f_{l, 1}\left(z_{1}\right) \sum_{k=0}^{4} f_{k, m}^{l}(z) Q_{l, k}\right), \quad 0 \leq z, z_{1} \leq 1 \text {, }
$$

where

$$
\left\{\begin{array}{lll}
Q_{1,0}=(1,0,4), & Q_{0,1}=(2,0,8), & Q_{0,2}=(4,0,8), \\
Q_{0,3}=(5,0,4), & Q_{0,4}=(6,0,8), & \\
Q_{0,1}=(1,6,4), & Q_{1,1}=(2,6,0), & Q_{1,2}=(4,6,0), \\
Q_{1,3}=(6,6,4), & Q_{1,4}=(7,6,2), &
\end{array}\right.
$$




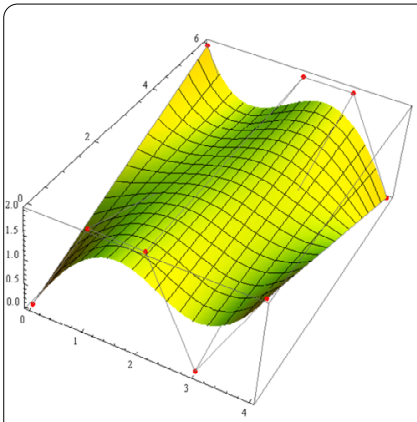

(a) $\mu, \nu, \mu_{1}, \nu_{1}=1$

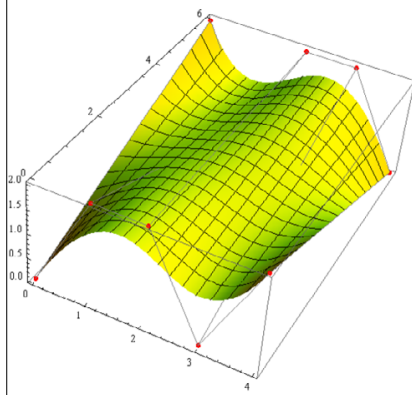

(d) $\mu, \nu=1, \mu_{1}, \nu_{1}=-1$

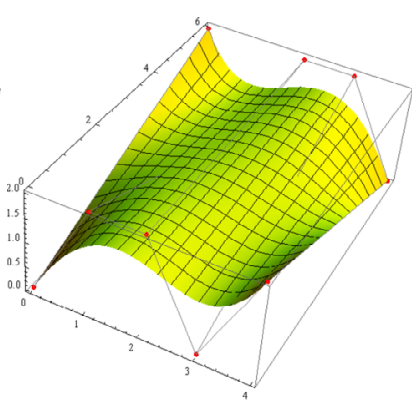

(b) $\mu, \nu, \mu_{1}, \nu_{1}=0.5$

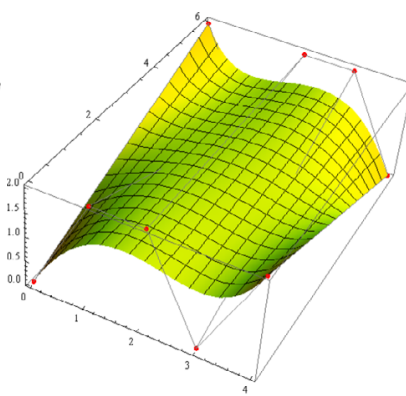

(e) $\mu, \nu=0, \mu_{1}, \nu_{1}=-1$

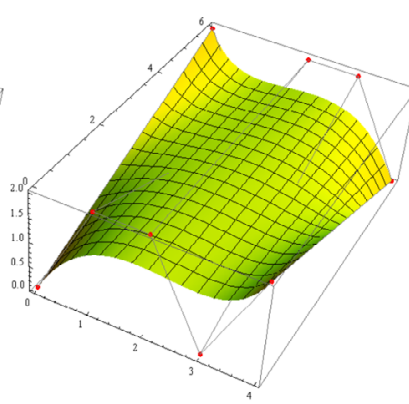

(c) $\mu, \nu, \mu_{1}, \nu_{1}=-0.5$

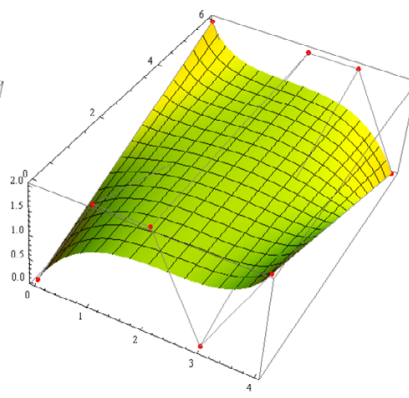

(f) $\mu, \nu, \mu_{1}, \nu_{1}=-1$

Figure 9 GBT-Bézier ruled surfaces with different shape parameters

are the control points of GLs. Figure 9 illustrates the GBT-Bézier ruled surfaces determined by (19) with various shape parameters.

Two adjacent GBT-Bézier ruled surfaces satisfying the $C^{1}$ and $C^{2}$ continuity in $z$ direction can be presented in Figs. 10 and 11 with third and fourth degree GBT-Bézier curves taken as GLs of the right and left GBT-Bézier ruled surface, respectively. Figure 12 shows the $C^{3}$ continuity of two adjacent GBT-Bézier ruled surfaces that have forth degree GBTBézier curves as GLs in $z$ direction.

\subsection{GBT-Bézier swung surface along shape parameters}

A swung surface can be generated from the surface of revolution [25]. Using a tensor product GBT-Bézier surface design with shape parameters, a swung surface is described in this portion. Let

$$
\left\{\begin{array}{l}
F_{m}(z, \mu, v)=\sum_{k=0}^{m} f_{k, m}(z) Q_{k, 0}, \quad 0 \leq z \leq 1, \\
F_{n}\left(z_{1}, \mu_{1}, v_{1}\right)=\sum_{l=0}^{n} f_{l, n}\left(z_{1}\right) Q_{1, l}, \quad 0 \leq z_{1} \leq 1,
\end{array}\right.
$$

be the two GBT-Bézier curves described by (3), where $Q_{k, 0}=\left(Q_{k, 0}^{X}, 0, Q_{k, 0}^{Z}\right)$ and $Q_{l, 1}=$ $\left(Q_{l, 1}^{X}, Q_{l, 1}^{Y}, 0\right)$ are their control points, and $F_{m}(z, \mu, v)$ and $F_{n}\left(z_{1}, \mu_{1}, \nu_{1}\right)$ are known as profile curves (PCs) and trajectory curves (TCs), respectively. They are described in the $X O Z$ plane and $X O Y$ plane, respectively. Vector form of the these curves and their corresponding nonzero coordinate functions $F_{m}^{X}(z), F_{m}^{Z}(z)$ and $F_{n}^{X}\left(z_{1}\right), F_{n}^{Y}\left(z_{1}\right)$ are used to construct a 


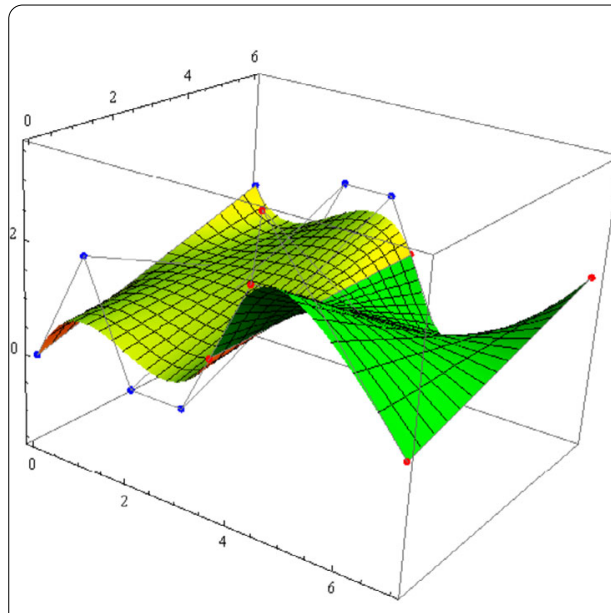

(a) $\mu, \nu_{1}=0.5, \mu_{1}, \nu=-0.01$

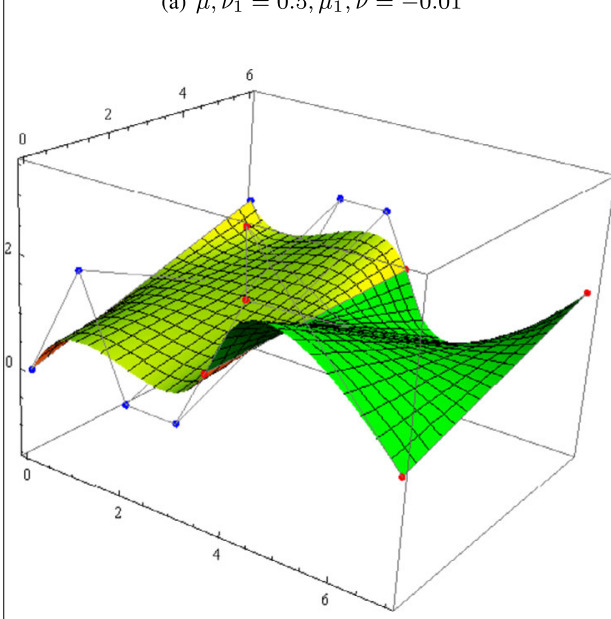

(c) $\mu, \nu_{1}=-1, \mu_{1}, \nu=0$

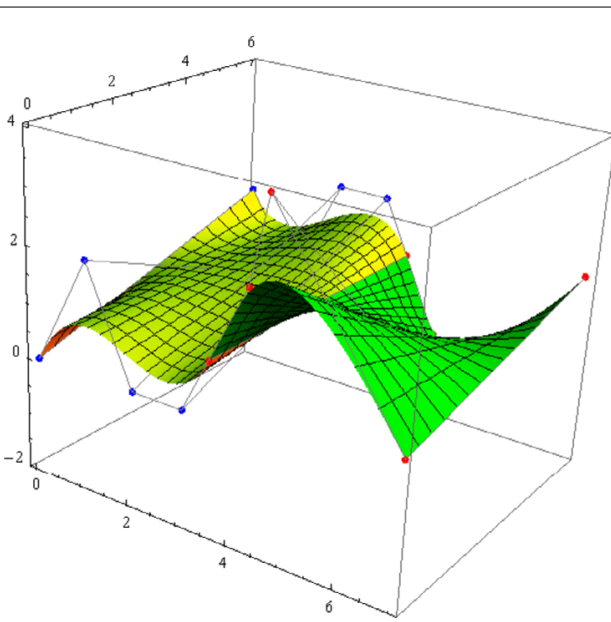

(b) $\mu, \nu_{1}=0.5, \mu_{1}, \nu=0$

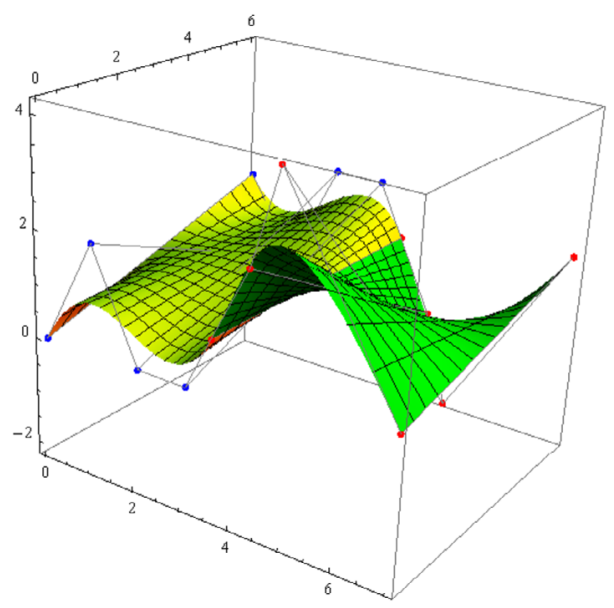

(d) $\mu, \nu_{1}=1, \mu_{1}, \nu=0$

Figure $10 C^{1}$ continuity between two GBT-Bézier ruled surfaces

swung surface given by

$$
\left\{\begin{array}{l}
F_{m}(z, \mu, v)=\left\{F_{m}^{X}(z), 0, F_{m}^{Z}(z)\right\}, \\
F_{n}\left(z_{1}, \mu_{1}, v_{1}\right)=\left\{F_{n}^{X}\left(z_{1}\right), F_{n}^{Y}\left(z_{1}\right), 0\right\},
\end{array}\right.
$$

and the GBT-Bézier swung surface is defined by $[25,26]$

$$
T_{\text {swung }}\left(z, z_{1}\right)=\left(\lambda F_{m}^{X}(z) F_{n}^{X}\left(z_{1}\right), \lambda F_{m}^{X}(z) F_{n}^{Y}\left(z_{1}\right), F_{m}^{Z}(z)\right) \text {, }
$$

where $\lambda(\lambda>0)$ is an arbitrary scaling factor.

Proposition 3.5 The GBT-Bézier swung surface expressed by (22) has the following geometric characteristics: if the profile curve $F_{m}(z)$ is swung about $z$-axis and re-scale in the $X$ and $Y$ directions, the swung surface $T_{\text {swung }}\left(z, z_{1}\right)$ is achieved in Fig. 13 with different values of scaling factor $\lambda$. 


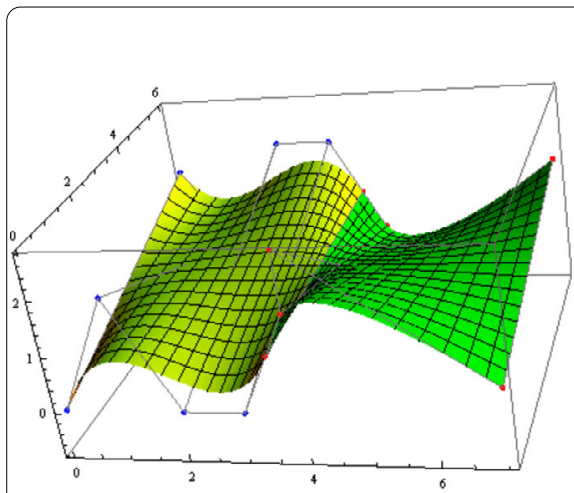

(a) $\mu, \nu=0.5, \mu_{1}, \nu_{1}=0.6$

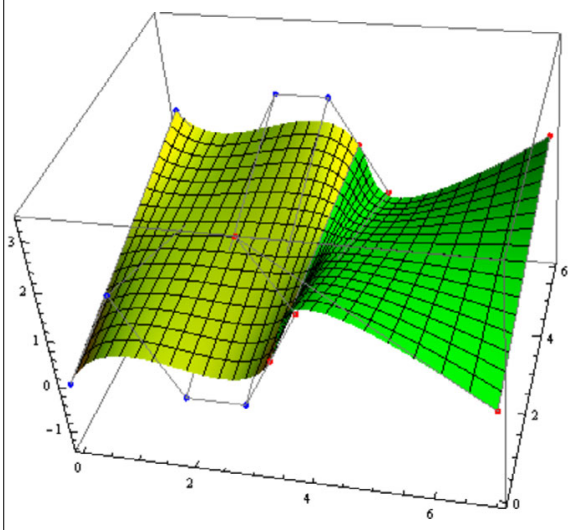

(c) $\mu, \nu=0.9, \mu 1, \nu_{1}=1$

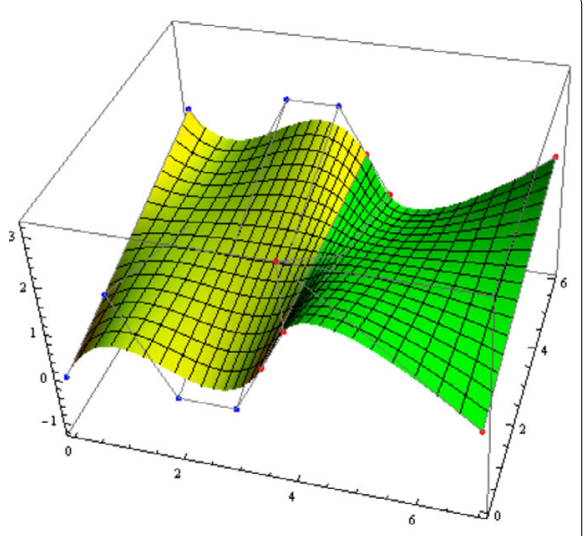

(b) $\mu, \nu=0.5, \mu_{1}, \nu_{1}=-0.2$

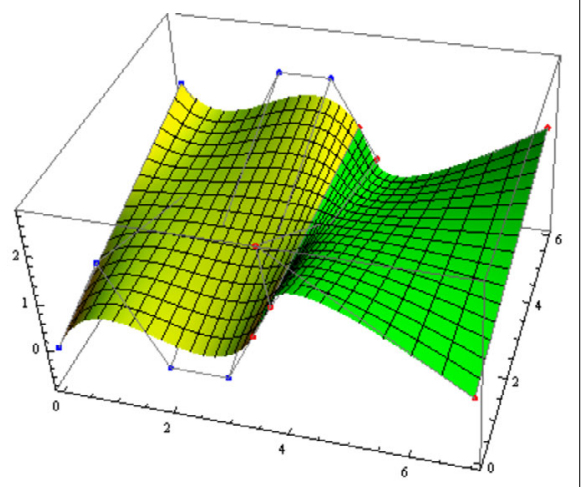

(d) $\mu, \nu=-0.5, \mu 1, \nu_{1}=1$

Figure $11 C^{2}$ continuity between two GBT-Bézier ruled surfaces

Proposition 3.6 Here, some properties of $z$ and $z_{1}$ lines of swung surfaces are as follows:

1. If the $z$ line is fixed, the curves $T_{\mathrm{swung}}\left(\bar{z}, z_{1}\right)$ which have identical trajectory curve $F_{n}\left(z_{1}\right)$ but are scaled as $\lambda F_{m}^{X}(z)$ in $X$ and $Y$ directions are produced.

2. The fixed $\bar{z}_{1}$ line produces curves $Z_{\text {swung }}\left(z, \bar{z}_{1}\right)$ with the same profile curve $F_{m}(z)$, and the connection between the curves $T_{\text {swung }}\left(z, \bar{z}_{1}\right)$ and the PCs $F_{m}(z)$ is: the resulting curves $T_{\text {swung }}\left(z, \bar{z}_{1}\right)$ are achieved by rotating the PCs $F_{m}(z)$ into the plane with the vector $\left(F_{n}^{X}\left(\bar{z}_{1}\right), F_{n}^{Y}\left(\bar{z}_{1}\right), 0\right)$, and use the scale factor $\lambda\left|F_{n}\left(z_{1}\right)\right|$ to scale the rotated curve along $X$ and $Y$ coordinates while $Z$ coordinate stays unscaled.

Theorem 4 The GBT-Bézier swung surface (22) can be converted into its tensor product form:

$$
T_{\text {swung }}\left(z, z_{1}, \mu, v, \mu_{1}, v_{1}\right)=\sum_{k=0}^{m} \sum_{l=0}^{n} Q_{k, l} f_{k, m}(z) f_{l, n}\left(z_{1}\right), \quad z, z_{1} \in[0,1],
$$

where $Q_{k, l}=\left(\lambda Q_{k, 0}^{X} Q_{l, 1}^{X}, \lambda Q_{k, 0}^{X} Q_{l, 1}^{Y}, Q_{k, 0}^{Z}\right), k=0,1, \ldots, m ; l=0,1, \ldots, n$, are the control net points of the GBT-Bézier swung surface, $Q_{k, 0}^{X}, Q_{k, 0}^{Z}$ and $Q_{l, 1}^{X}, Q_{k, 1}^{Y}$ are elements of the control points $Q_{k, 0}$ and $Q_{l, 1}$. The shapes of profile and trajectory curve are controlled by the shape parameters $\mu, v$ and $\mu_{1}, v_{1}$, respectively. 


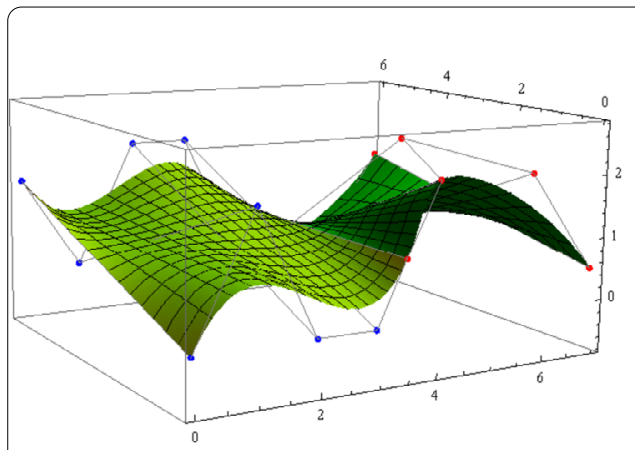

(a) $\mu=1, \mu_{1}=-0.7, \nu=-0.5, \nu_{1}=0.6$

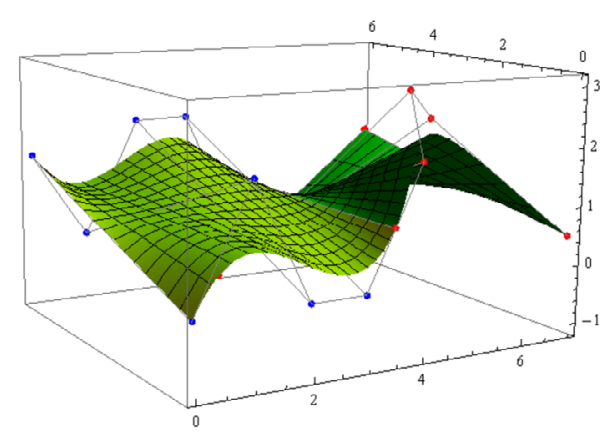

(c) $\mu=1, \mu_{1}, \nu=-0.4, \nu_{1}=0.6$

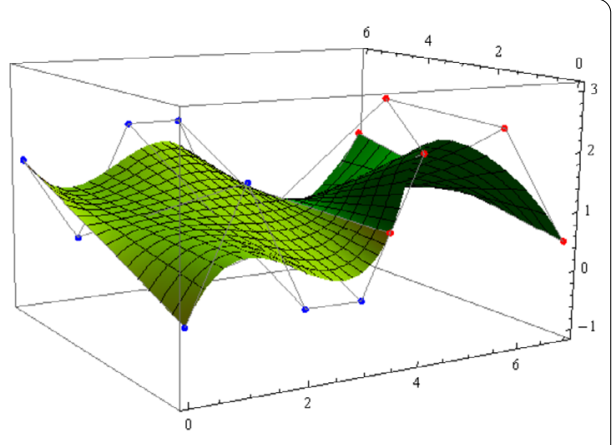

(b) $\mu=1, \mu_{1}=-0.7, \nu=-0.4, \nu_{1}=0.6$

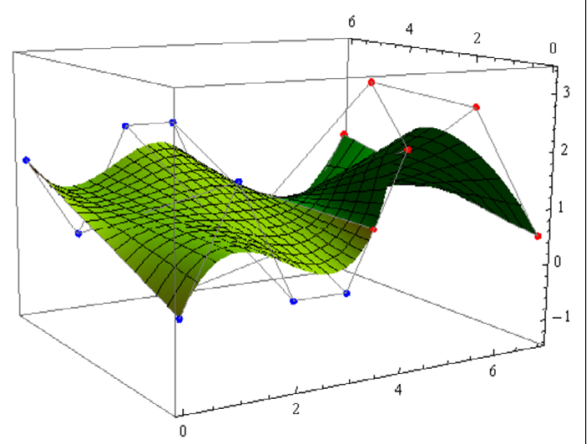

(d) $\mu=1, \mu_{1}=-0.7, \nu=-0.3, \nu_{1}=0.6$

Figure $12 C^{3}$ continuity between two GBT-Bézier ruled surfaces

Proof From equations (20) and (21) of swung surface, we have

$$
\left\{\begin{array}{l}
F_{m}^{X}(z)=\sum_{k=0}^{m} Q_{k, 0}^{X} f_{k, m}(z), F_{m}^{Z}(z)=\sum_{k=0}^{m} Q_{k, 0}^{Z} f_{k, m}(z), \\
F_{n}^{X}\left(z_{1}\right)=\sum_{l=0}^{n} Q_{l, 1}^{X} f_{l, n}\left(z_{1}\right), F_{n}^{Y}\left(z_{1}\right)=\sum_{l=0}^{n} Q_{l, 1}^{Y} f_{l, n}\left(z_{1}\right) .
\end{array}\right.
$$

Now, substituting equation (24) into equation (22) and using the partition of unity property $\sum_{k=0}^{m} f_{k, m}(z)=1$, we have

$$
\begin{aligned}
& T_{\text {swung }}\left(z, z_{1}\right) \\
&=\left(\lambda F_{m}^{X}(z) F_{n}^{X}\left(z_{1}\right), \lambda F_{m}^{X}(z) F_{n}^{Y}\left(z_{1}\right), F_{m}^{Z}(z)\right) \\
&=\left(\lambda \sum_{k=0}^{m} Q_{k, 0}^{X} f_{k, m}(z) \sum_{l=0}^{n} Q_{l, 0}^{X} f_{l, n}\left(z_{1}\right), \lambda \sum_{k=0}^{m} Q_{k, 0}^{X} f_{k, m}(z) \sum_{l=0}^{n} Q_{l, 0}^{Y} f_{l, n}\left(z_{1}\right), \sum_{k=0}^{m} Q_{k, 0}^{Z} f_{k, m}(z)\right) \\
&=\left(\sum_{k=0}^{m} \sum_{l=0}^{n} f_{k, m}(z) f_{l, n}\left(z_{1}\right)\left(\lambda Q_{k, 0}^{X} Q_{l, 0}^{X}\right), \sum_{k=0}^{m} \sum_{l=0}^{n} f_{k, m}(z) f_{l, n}\left(z_{1}\right)\left(\lambda Q_{k, 0}^{X} Q_{l, 0}^{Y}\right)\right. \\
&\left.\sum_{k=0}^{m} \sum_{l=0}^{n} f_{k, m}(z) f_{l, n}\left(z_{1}\right) Q_{k, 0}^{z}\right) \\
&= \sum_{k=0}^{m} \sum_{l=0}^{n} f_{k, m}(z) f_{l, n}\left(z_{1}\right)\left(\lambda Q_{k, 0}^{X} Q_{l, 0}^{X}, \lambda Q_{k, 0}^{X} Q_{l, 0}^{Y}, Q_{k, 0}^{Z}\right)
\end{aligned}
$$


Thus, a tensor product GBT-Bézier surface design of degree $(n, m)$ is used to express the swung surfaces (22).

The swung surface described by Theorem 4 is called GBT-Bézier swung surface. It appears that the GBT-Bézier swung surface carried all the characteristics and benefits of GBT-Bézier surface. The shape parameters are used to modify GBT-Bézier swung surface by keeping control net points fixed. For smooth connection among adjacent GBT-Bézier swung surfaces like previous surfaces, if the PCs or the TCs are combined by $C^{1}$ or $C^{2}$ continuity for GBT-Bézier curve, then the resulting GBT-Bézier swung surface is also a $C^{1}$ or $C^{2}$ continuous surface in the direction of profile or trajectory curve. It is worth noting that if the swung surface $T_{\text {swung }}\left(z, z_{1}\right)$ is either open or close in $z$ and $z_{1}$ directions, then the corresponding profile curve $F_{m}(z)$ and the trajectory curve $F_{n}\left(z_{1}\right)$ both will be either open or closed. Additionally, if the scale factor $\lambda=1$ and the trajectory curve $F_{n}\left(z_{1}\right)$ circle with a unit radius with a center at the origin, then the GBT-Bézier swung surface is a surface of revolution. However, the surface of revolution no longer is a tensor product GBT-Bézier swung surface which is described by Theorem 4.

The detailed algorithm for designing a GBT-Bézier swung surface is described as follows.

\section{Algorithm 4}

1. Take a profile curve in $X O Z$ and a trajectory curve in the $X O Y$ plane in the form of GBT-Bézier curves.

2. Define a GBT-Bézier swung surface by using equation (23).

3. Take the control net points of the GBT-Bézier swung surface in the pattern $Q_{k, l}=\left(\lambda Q_{k, 0}^{X} Q_{l, 1}^{X}, \lambda Q_{k, 0}^{X} Q_{l, 1}^{Y}, Q_{k, 0}^{Z}\right), k=0,1, \ldots, m ; l=0,1, \ldots, n$, where $Q_{k, 0}^{X}, Q_{k, 0}^{Z}$ and $Q_{l, 1}^{X}, Q_{k, 1}^{Y}$ are elements of the control points $Q_{k, 0}$ and $Q_{l, 1}$ described in expression (20).

4. Construct the GBT-Bézier swung surfaces in Wolfram Mathematica software using suitable values of scaling factor $\lambda$ and shape control parameters $\mu, \nu, \mu_{1}$, and $\nu_{1}$

5. Two adjacent GBT-Bézier swung surfaces can be connected by $C^{1}$ and $C^{2}$ continuity conditions given in Theorem 2.

Example 3.4 Figures 13-15 show the GBT-Bézier swung surfaces for the different values of scale factor and shape parameters. In these figures, the PCs $F_{m}(z)$ and the TCs $F_{n}\left(z_{1}\right)$ are both GBT-Bézier curves of degree three and the mesh points are

$$
\begin{cases}Q_{0,0}=(3,0,5), & Q_{1,0}=(1,0,3), \\ Q_{2,0}=(1,0,2), & Q_{3,0}=(4,0,1), \\ Q_{0,1}=(4,1,0), & Q_{1,1}=(1,2,0), \\ Q_{2,1}=(1,4,0), & Q_{3,1}=(5,5,0) .\end{cases}
$$

It can be observed from Fig. 13 that the scaling of swung surfaces in both directions is handled by scale factor $\lambda$ keeping shape parameters $\mu$ and $v$ same and fixed. Figures 14 and 15 exhibit the graphs of GBT-Bézier swung surfaces along the same control points of the PCs and TCs as taken in Fig. 13, but the values of shape parameters are different and scaling factor $\lambda=0.15$ is fixed. Figures 16,17 , and 18 depict the $C^{1}$ and $C^{2}$ continuity connection of two adjacent GBT-Bézier swung surfaces for various values of shape control parameters and scaling factor $\lambda$. 


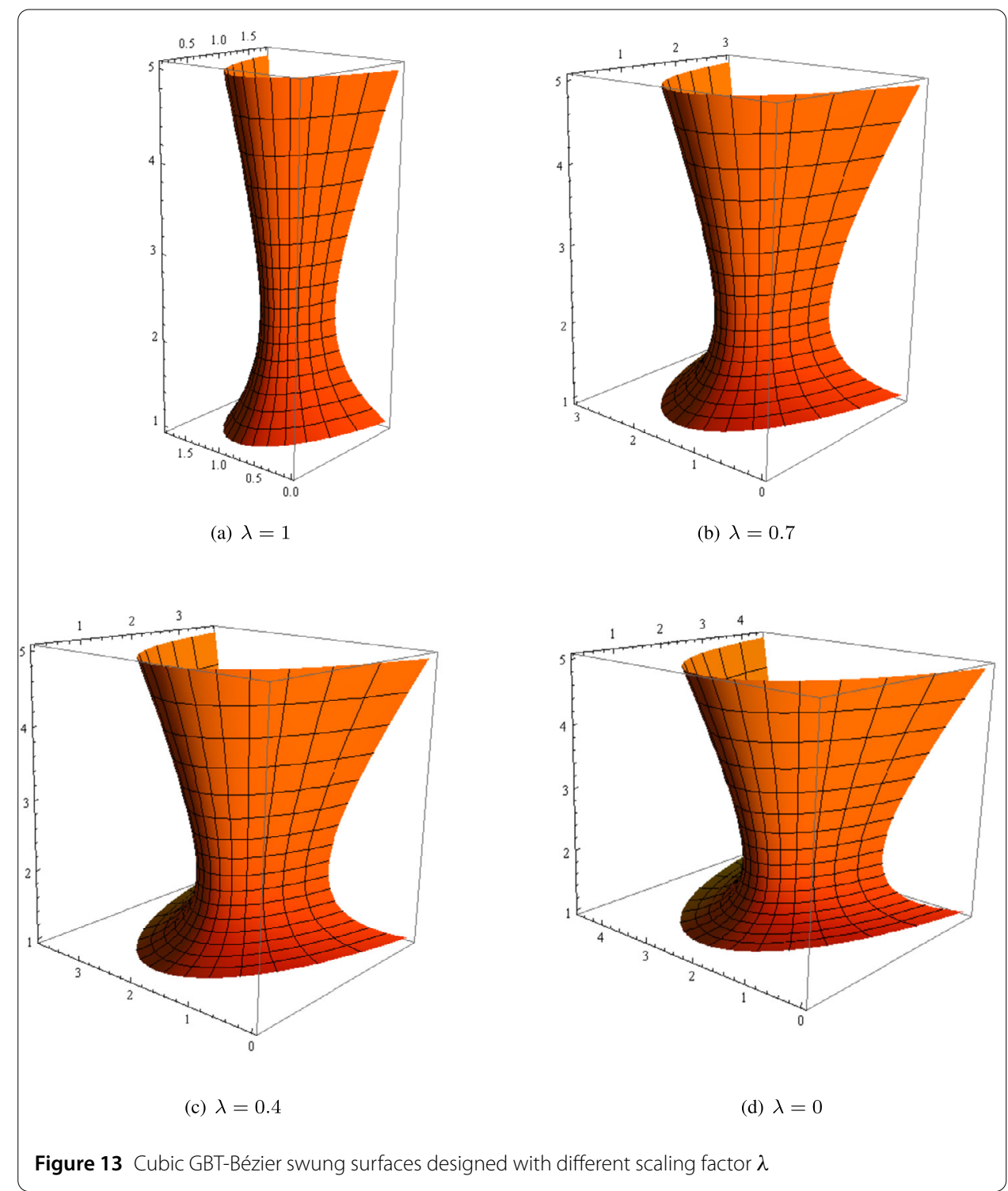

\subsection{GBT-Bézier swept surfaces with shape parameters}

In this section, we especially tackle the problem of constructing a surface by sweeping a section curve (SC) around an arbitrary TC [11]. The GBT-Bézier curves of order $m$ and $n$ are taken as $\mathrm{SCs}$ and TCs in a $3 D$ space, respectively. These two curves are given by

$$
\left\{\begin{array}{l}
F_{m}(z ; \mu, v)=\sum_{k=0}^{m} f_{k, m}(z) Q_{k, m}, \quad 0 \leq z \leq 1, \\
F_{n}\left(z_{1} ; \mu_{1}, v_{1}\right)=\sum_{l=0}^{n} f_{l, n}\left(z_{1}\right) Q_{l, n}, \quad 0 \leq z_{1} \leq 1 .
\end{array}\right.
$$

Generally, a swept surface is given by [25]

$$
T_{\text {swept }}\left(z, z_{1} ; \mu, v, \mu_{1}, v_{1}\right)=F_{n}\left(z_{1} ; \mu_{1}, v_{1}\right)+\mathbf{S}\left(z_{1}\right) F_{m}(z ; \mu, \nu),
$$

where $\mathbf{S}\left(z_{1}\right)$ is a $3 \times 3$ matrix integrating scaling and rotation of the $\mathrm{SC} F_{m}(z)$. In this study, the SC $F_{m}(z)$ and the TCs $F_{n}\left(z_{1}\right)$ both are GBT-Bézier curves, but the type and shape might 


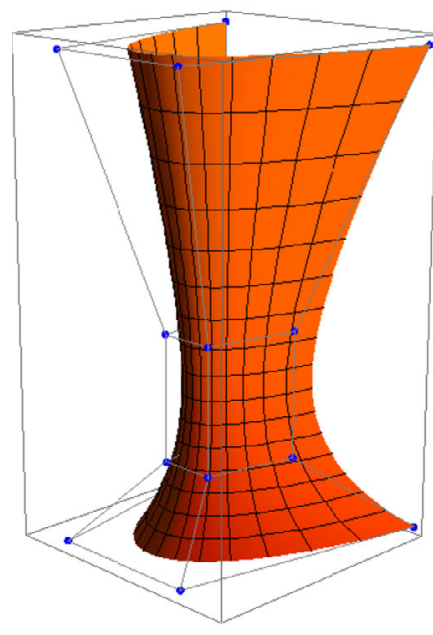

(a) $\mu=1, \nu=1$

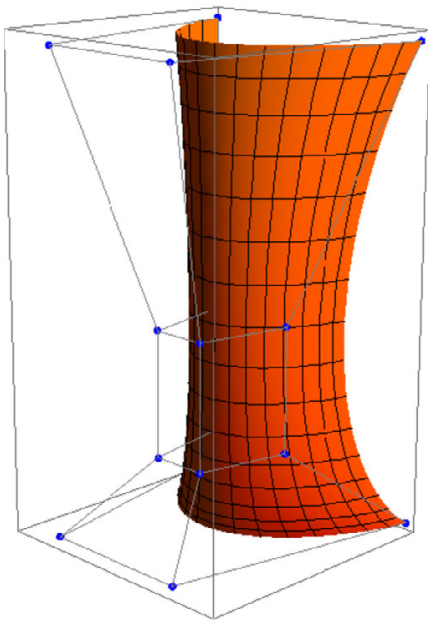

(c) $\mu=0, \nu=1$

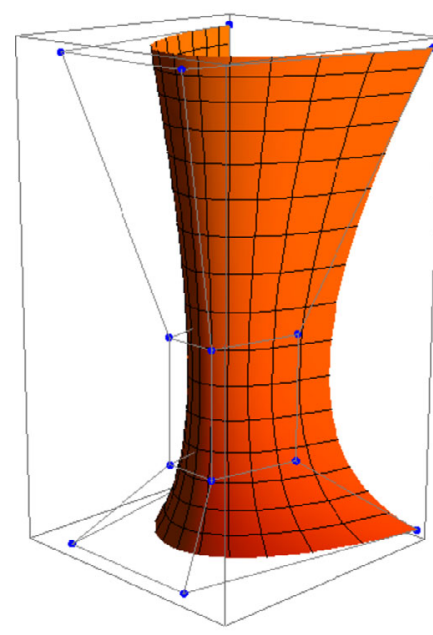

(b) $\mu=0.5, \nu=1$

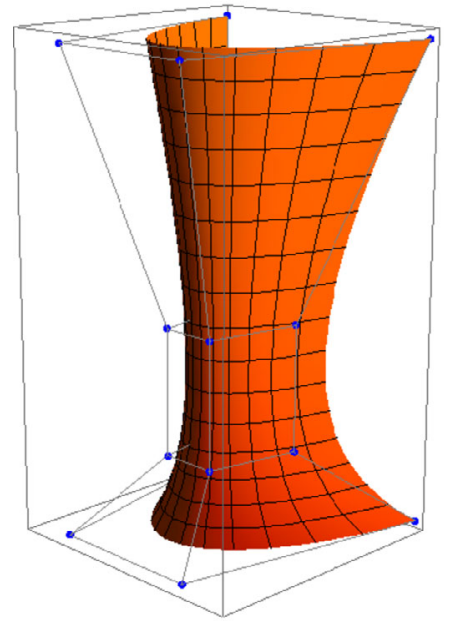

(d) $\mu=-1, \nu=1$

Figure 14 GBT-Bézier swung surfaces with different shape parameter $\mu$

be random (single or composite, planar or non-planar, open or closed). Normally, equation (28) can generate an unnecessary surface with degeneracy and without continuity. Moreover, in many situations the swept surface $T_{\text {swept }}\left(z, z_{1}\right)$ is not exactly expressible as a surface model existing in available literature such as B-spline, Bézier, and NURBS surfaces. Practically, swept surfaces can be generated by the following two categories:

- $\mathbf{M}\left(z_{1}\right)$ is an identity matrix, which for all $z_{1}$ and $F_{m}(z)$ is just translated by $F_{n}\left(z_{1}\right)$.

- $\mathbf{M}\left(z_{1}\right)$ is not an identity matrix.

The second type is very complicated and debatable, which can be referred to literature [25]. Particularly, we consider the first case of swept surfaces which can be defined by

$$
T_{\text {swept }}\left(z, z_{1} ; \mu, \nu, \mu_{1}, v_{1}\right)=F_{n}\left(z_{1}\right)+F_{m}(z) \text {. }
$$




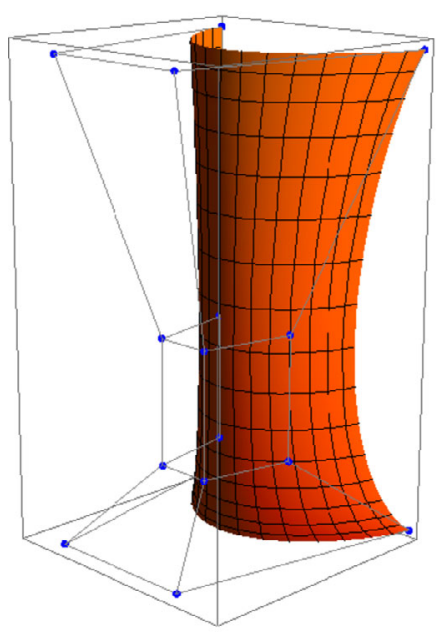

(a) $\mu=0.5, \nu=1$

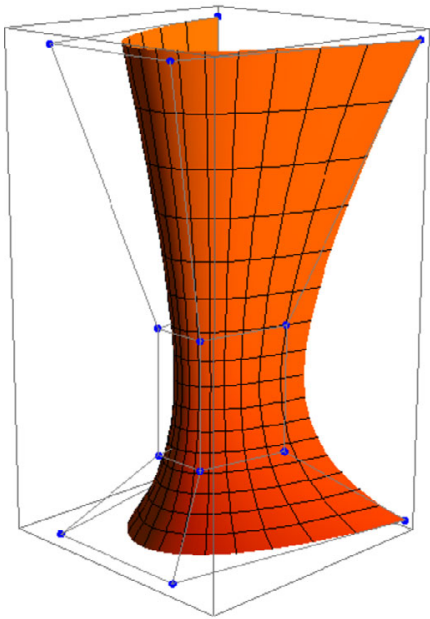

(c) $\mu=0.5, \nu=0.4$

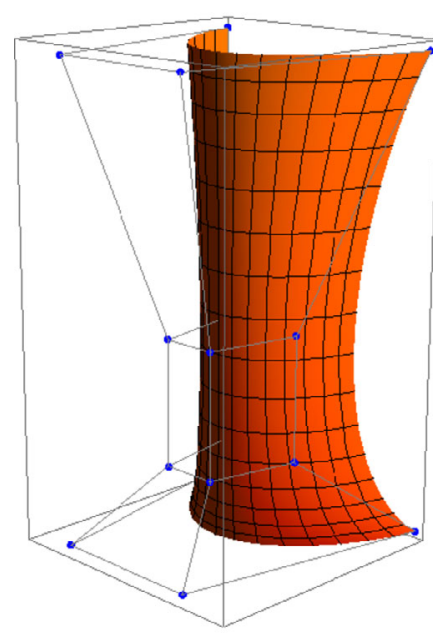

(b) $\mu=0.5, \nu=0.7$

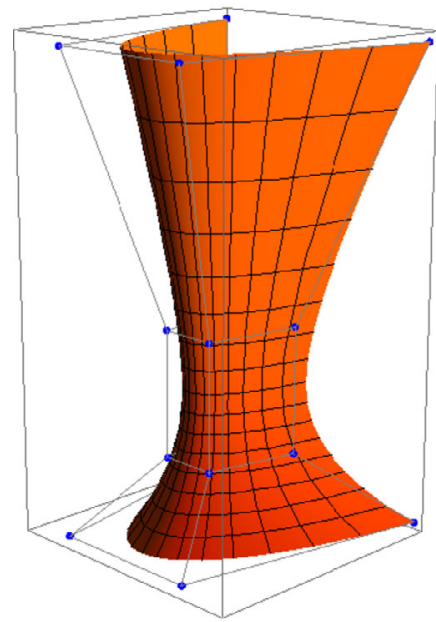

(d) $\mu=0.5, \nu=0$

Figure 15 GBT-Bézier swung surfaces with various shape parameter $v$

The curves $F_{m}(z)$ and $F_{n}\left(z_{1}\right)$ are GBT-Bézier curves described by (27). The surface (29) is termed as the GBT-Bézier swept surface.

Theorem 5 A GBT-Bézier swept surface (29) can be expressed in its tensor product form:

$$
T_{\text {swept }}\left(z, z_{1}, \mu, v, \mu_{1}, v_{1}\right)=\sum_{k=0}^{m} \sum_{l=0}^{n} f_{k, m}(z) f_{l, n}\left(z_{1}\right) \widehat{Q}_{k, l}, \quad z, z_{1} \in[0,1]
$$

where $\widehat{Q}_{k, l}=Q_{k, m}+Q_{l, n}(k=0,1, \ldots, m ; l=0,1, \ldots, n)$ are the control net points. 


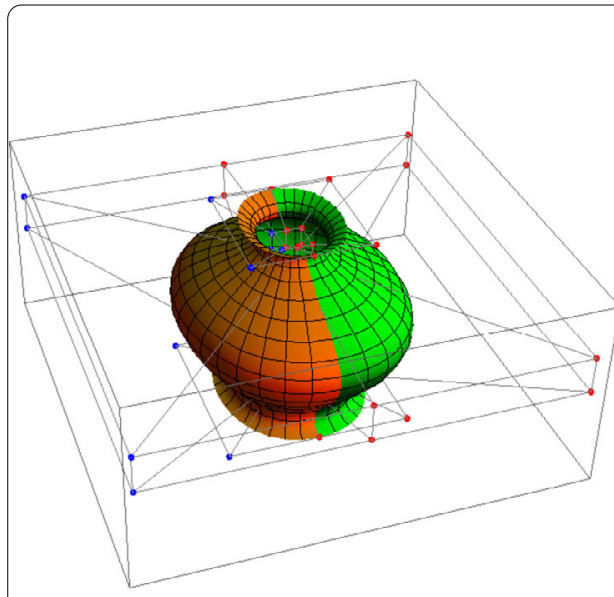

(a) $\lambda=0.4, \forall \mu, \nu=0.5$

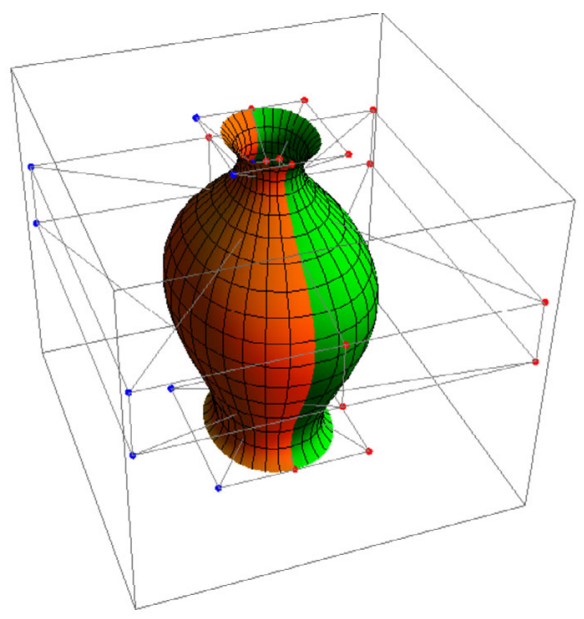

(c) $\lambda=0.2, \forall \mu, \nu=0.5$

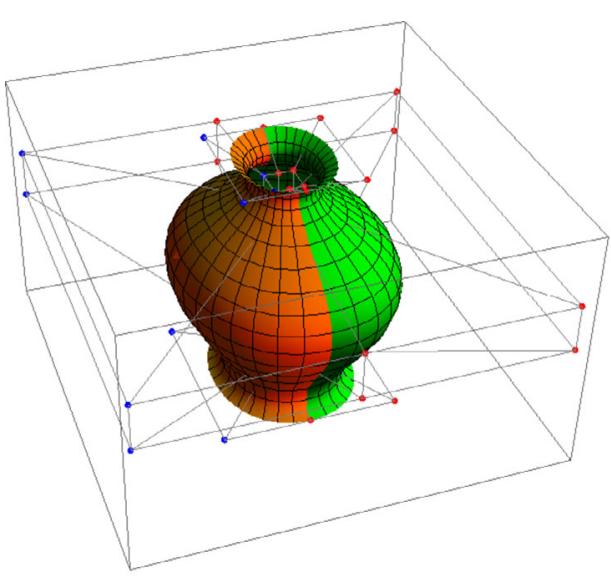

(b) $\lambda=0.3, \forall \mu, \nu=0.5$

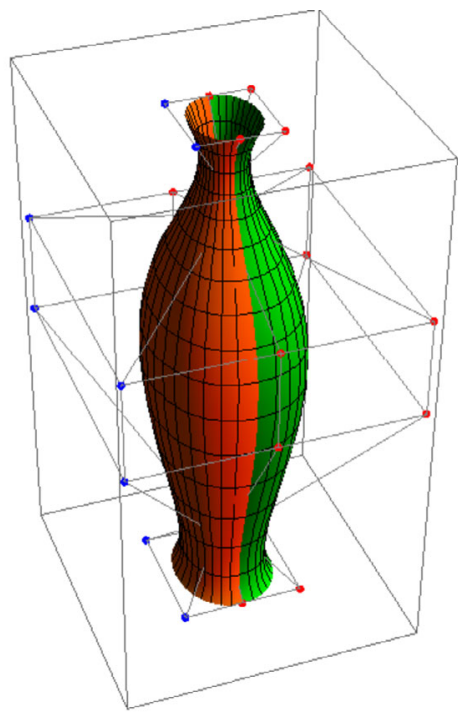

(d) $\lambda=0.1, \forall \mu, \nu=0.5$

Figure $16 C^{1}$ continuous connection among two GBT-Bézier swung surfaces with different scale factor $\lambda$

Proof From the definition of GBT-Bézier swept surface, and substituting equation (27) into equation (29), we have

$$
\begin{aligned}
T_{\text {swept }}\left(z, z_{1} ; \mu, \nu, \mu_{1}, v_{1}\right) & =F_{n}\left(z_{1}\right)+F_{m}(z) \\
& =\sum_{l=0}^{n} Q_{l, n} f_{l, n}\left(z_{1}\right)+\sum_{k=0}^{m} Q_{k, m} f_{k, m}(z) \\
& =\sum_{k=0}^{m} f_{k, m}(z) \sum_{l=0}^{n} Q_{l, n} f_{l, n}\left(z_{1}\right)+\sum_{l=0}^{n} f_{l, n}\left(z_{1}\right) \sum_{k=0}^{m} Q_{k, m} f_{k, m}(z) \\
& =\sum_{k=0}^{m} \sum_{l=0}^{n} f_{k, m}(z) f_{l, n}\left(z_{1}\right) Q_{l, n}+\sum_{k=0}^{m} \sum_{l=0}^{n} f_{k, m}(z) f_{l, n}\left(z_{1}\right) Q_{k, m}
\end{aligned}
$$




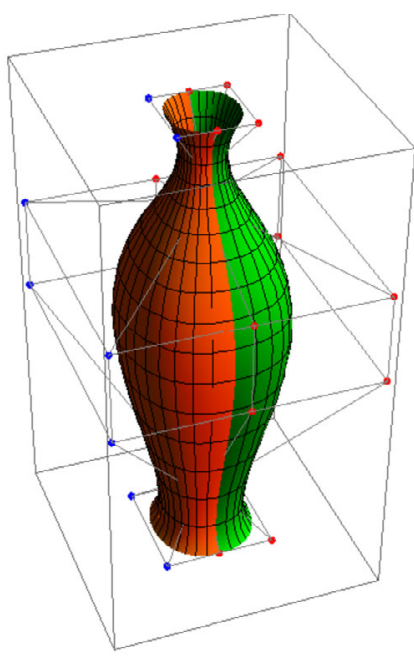

(a) $\lambda=0.1, \mu, \nu=1$

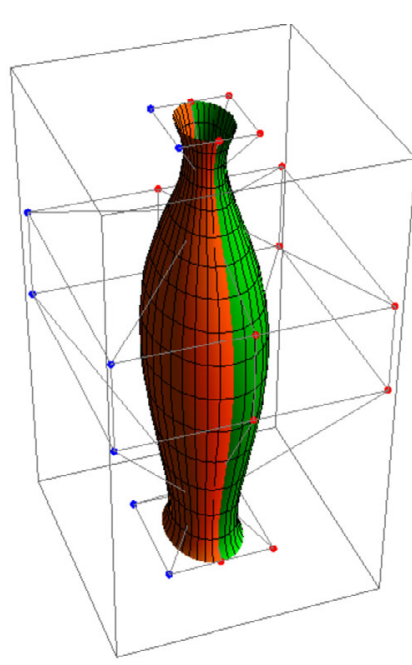

(b) $\lambda=0.1, \mu, \nu=0$

Figure $17 C^{1}$ continuous connection among two GBT-Bézier swung surfaces

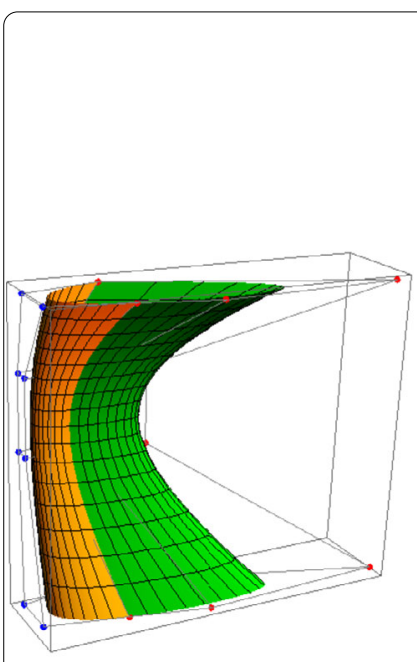

(a) $\forall \mu, \nu=0.5$

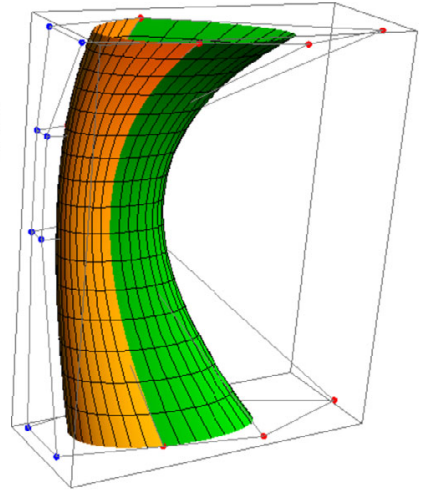

(b) $\forall \mu, \nu=0$

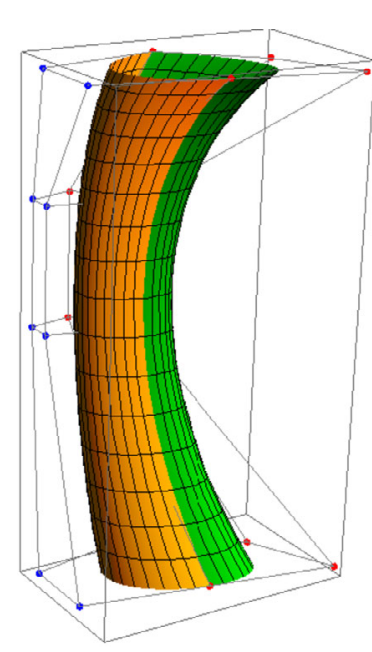

(c) $\forall \mu, \nu=-0.5$

Figure $18 C^{2}$ continuity between two GBT-Bézier swung surfaces with $\lambda=0.7$

$$
\begin{aligned}
& =\sum_{k=0}^{m} \sum_{l=0}^{n} f_{k, m}(z) f_{l, n}\left(z_{1}\right)\left(Q_{l, n}+Q_{k, m}\right) \\
& =\sum_{k=0}^{m} \sum_{l=0}^{n} f_{k, m}(z) f_{l, n}\left(z_{1}\right) \widehat{Q}_{k, l} .
\end{aligned}
$$

Thus, the GBT-Bézier swept surface described by (29) can be exactly expressed as tensor product GBT-Bézier surface design.

Proposition 3.7 Equation (30) can be demonstrated: 
1. As the tensor product GBT-Bézier surface is used to express the GBT-Bézier swept surface, so it holds all the characteristics and benefits of the GBT-Bézier surface.

2. By keeping control mesh fixed and varying the values of shape control parameters, the shape of the GBT-Bézier swept surface can be modified. Furthermore, the composite GBT-Bézier swept surfaces can also be constructed like the previous surfaces.

A GBT-Bézier swept surface can be constructed as follows:

\section{Algorithm 5}

1. Consider a SC which sweeps to the trajectory curve (both curves in the form of GBT-Bézier curves).

2. Construct the GBT-Bézier swept surface by using equation (30).

3. Follow the pattern $\widehat{Q}_{k, l}=Q_{k, m}+Q_{l, n}(k=0,1, \ldots, m ; l=0,1, \ldots, n)$ and take the control points of GBT-Bézier swept surface where $Q_{k, m}, Q_{l, n}$ are the control points of section and TCs, respectively.

4. Draw GBT-Bézier swept surfaces in Wolfram Mathematica software by taking different values of shape control parameters $\mu, v$ in their respective value range $[-1,1]$.

5. Two adjacent GBT-Bézier swept surfaces can be connected by $C^{1}$ and $C^{2}$ continuity conditions given in Theorem 2.

Example 3.5 The shape control parameters of the two contiguous curves segments are used $\mu, \mu_{1}, v, v_{1}$ and $\mu_{2}, \mu_{3}, v_{2}, v_{3}$, respectively, and these values are identical for two SCs as well as are similar for two TCs case. The mesh points are taken as $(2,0,2),(1,0,3)$, $(5,0,4),(6,0,3)$ and $(6,0,3),(7,0,2),(3,0,1),(2,0,2)$. The control points of the two TCs are taken as $(4,0,0),(0,1,0),(0,2,0),(4,3,0)$ and $(4,3,0),(8,4,0),(8,5,0),(4,6,0)$. Figure 19 and Fig. 20 depict the composite curves of two contiguous GBT-Bézier swept surfaces with different shape parameters which can be connected by $C^{1}$ and $C^{2}$ continuity constraints, respectively.

\subsection{GBT-Bézier rotation surfaces with shape parameters}

Geometric shapes of many objects are rotation surfaces in nature. Rotation surface is a type of special and common geometric profile in the field of product modeling design and has a broad demand in many fields such as aeronautic, architectural engineering, industrial designing, machining, and computer graphics. In CAD/CAM technology, how we can obtain $3 D$ mathematical designs of rotation surfaces efficiently and swiftly is an important research issue. This portion is especially focused on how to develop a $C^{1}$ or $C^{2}$ continuous $3 D$ mathematical model of GBT-Bézier rotation surface by taking a combined GBT-Bézier curve. Rotation surfaces are created by revolving a curve along a straight line in space with a constant angle. When the GBT-Bézier curves $F_{m}(z ; \mu, v)(0<z \leq 1)$ are considered as the generating line and $\phi(0<\phi \leq 2 \pi)$ as a fixed angle around the rotation axis, then a rotation surface $R_{\text {rotation }}$ can be constructed in the $X O Z$ plane. Let us imagine that the generating line is a GBT-Bézier curve $F_{m}(z ; \mu, v)(0<z \leq 1)$ in the $X O Z$ plane and has $Z$-axis as the rotation axis, then the expression for rotation surface $R_{\text {rotation }}$ is described 


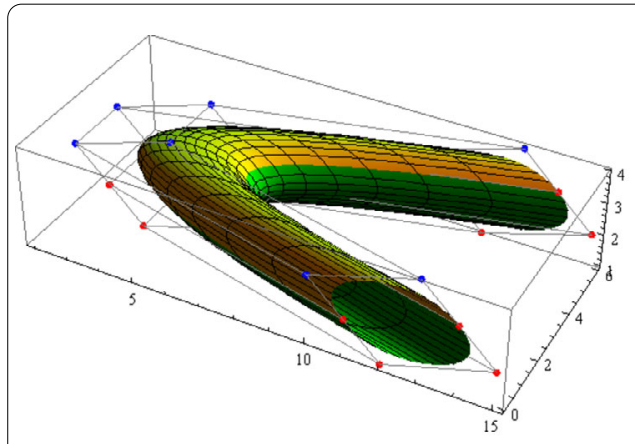

(a) $\forall \mu, \nu=1$

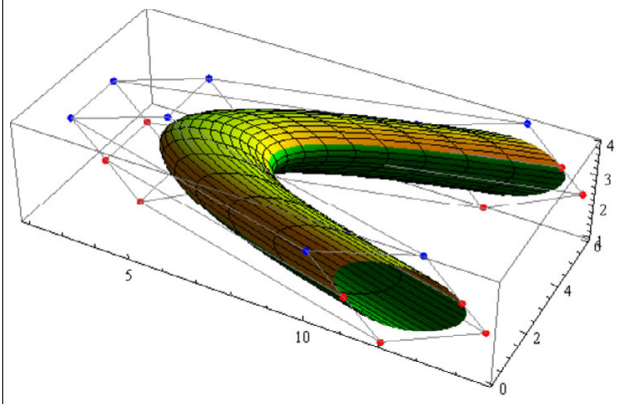

(c) $\forall \mu=1, \nu=0$

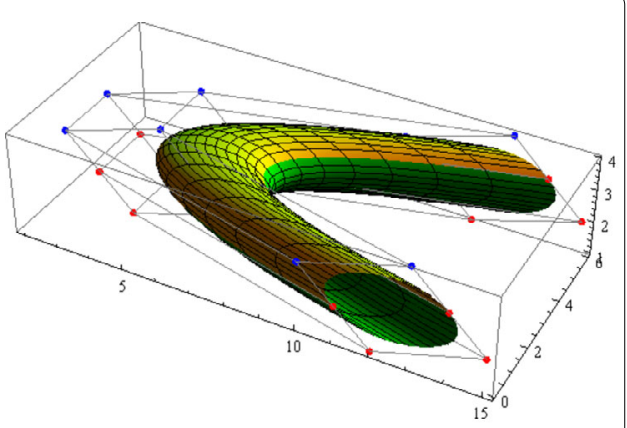

(b) $\forall \mu, \nu=0.5$

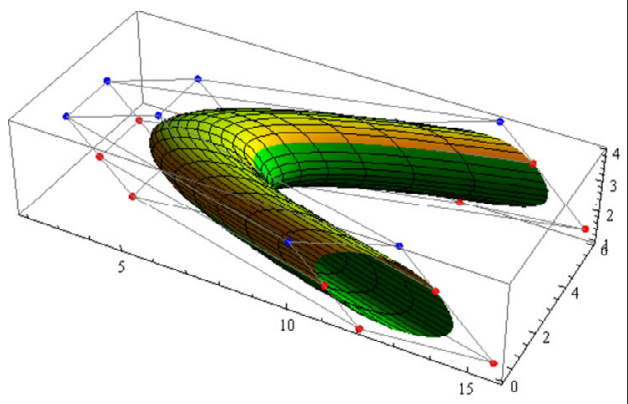

(d) $\forall \mu=0, \nu=1$

Figure $19 C^{1}$ continuity of two contiguous GBT-Bézier swept surfaces

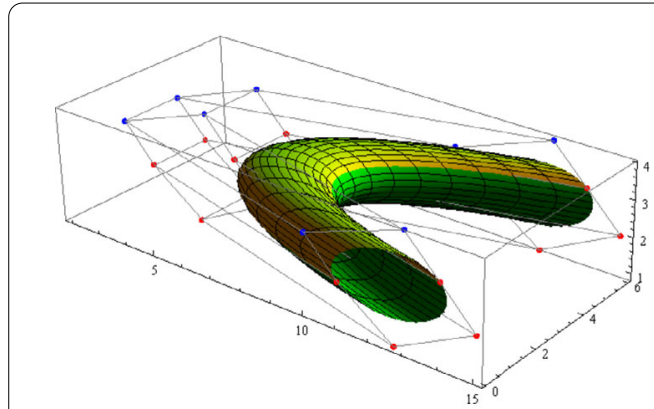

(a) $\mu, \mu_{1}, \mu_{3}=0, \mu_{2}, \nu, \nu_{2}=-0.3, \nu_{1}, \nu_{3}=-0.5$

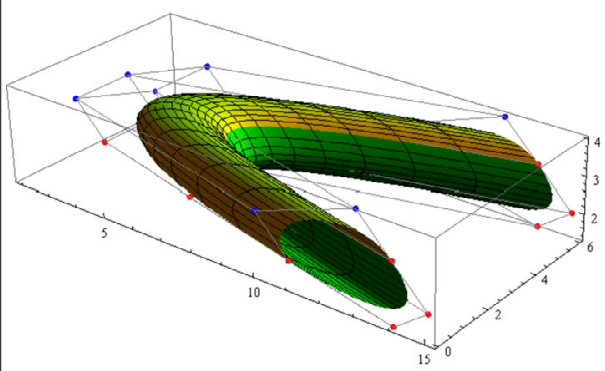

(c) $\mu_{2}, \nu, \nu_{2}=-0.3, \mu_{1}, \mu_{3}, \nu_{1}, \nu_{3}=1$

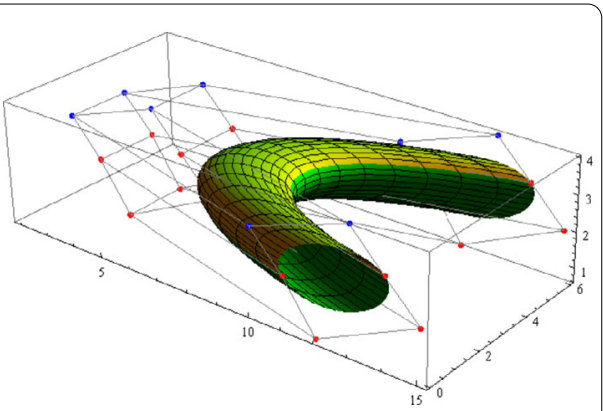

(b) $\mu_{2}, \nu, \nu_{2}=-0.3, \mu, \mu_{1}, \mu_{3}, \nu_{1}, \nu_{3}=-0.5$

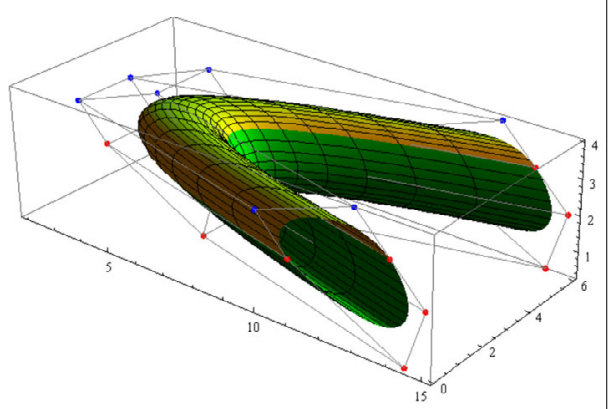

(d) $\mu_{2}, \nu, \nu_{2}=0, \mu, \mu_{1}, \mu_{3}, \nu_{1}, \nu_{3}=1$

Figure $\mathbf{2 0} C^{2}$ continuous GBT-Bézier swept surfaces with different shape parameters 
as follows [25]:

$$
\begin{aligned}
R_{\text {rotation }}(z, \phi) & =\left(\begin{array}{ccc}
\cos \phi & -\sin \phi & 0 \\
\sin \phi & \cos z \phi & 0 \\
0 & 0 & 1
\end{array}\right) F_{m}(z) \\
& =\left[X_{m}(z) \cos \phi, X_{m}(z) \sin \phi, Z_{m}(z)\right]^{T},
\end{aligned}
$$

where $0<\phi \leq 2 \pi$, the generating line $F_{m}(z)=\left[X_{m}(z), 0, Z_{m}(z)\right]^{T}, z \in[0,1]$. However, the rotation surface expressed by equation (32) comprises trigonometric functions that will enhance the mathematical complication and rounding error in developing a rotation surface. Practically, architects often desire to modify the shape of a rotating surface automatically and rapidly. Therefore, from the technique in [26], we define a class of GBT-Bézier rotation surfaces by taking the GBT-Bézier curves with their generatrices.

Theorem 6 Suppose that a GBT-Bézier curve $F_{m}(z)$ can be expressed by a variety of control points $Q_{k}(k=0,1, \ldots, m)$ whose coordinates are taken as $\left\{X_{k}, 0, Z_{k}\right\}(k=0,1, \ldots, m)$ in the $X O Z$ plane, then the expression for entire rotation surfaces generated by rotating the generating line $F_{m}(z)$ around $Z$-axis in one rotation is described as

$$
\left\{\begin{array}{l}
T_{\text {rotation }}^{Z}\left(z, z_{1}, \mu, v\right)=\left\{\frac{1-2 z_{1}}{2 z_{1}^{2}-2 z_{1}+1} X_{m}(z), \frac{2 z_{1}-2 z_{1}^{2}}{2 z_{1}^{2}-2 z_{1}+1} X_{m}(z), Z_{m}(z)\right\}, \\
\widetilde{T}_{\text {rotation }}^{Z}\left(z, z_{1}, \mu, v\right)=\left\{\frac{1-2 z_{1}}{2 z_{1}^{2}-2 z_{1}+1} X_{m}(z),-\frac{2 z_{1}-2 z_{1}^{2}}{2 z_{1}^{2}-2 z_{1}+1} X_{m}(z), Z_{m}(z)\right\},
\end{array}\right.
$$

where $T_{\text {rotation }}^{Z}$ and $\widetilde{T}_{\text {rotation }}^{Z}$ are symmetric about the XOZ plane, and by combining these two terms, we can get a complete GBT-Bézier rotation surface. The $X_{m}(z)$ and $Z_{m}(z)$ components of generating line $F_{m}(z)$ can be derived from the following expression:

$$
\left\{\begin{array}{l}
X_{m}(z)=\sum_{k=0}^{m} f_{k, m}(z) X_{k}, \quad z \in[0,1], \\
Z_{m}(z)=\sum_{l=0}^{m} f_{l, m}(z) Z_{l}, \quad z \in[0,1],
\end{array}\right.
$$

where $f_{k, m}(z)$ are GBTB degree $m$ given in equation (2).

Proof The proof of the above results given in Theorem 6 is as described in [21].

Conclusively, using the same results, we can develop a rotation surface if the generating line and the rotation axis are $X O Y$ or $Y O Z$ and $X$-axis or $Y$-axis, respectively.

Proposition 3.8 If the generating line $F_{m}(z)$ is revolved around the Z-axis with a fixed angle $\phi(0<\phi \leq 2 \pi)$, then the resulting surface is partisan GBT-Bézier rotation surface and is defined as follows:

(a) $T_{\text {rotation }}^{Z}$ in (33) is used to create the partially rotation surface if $0<\phi \leq \pi$, where $\phi$ is the rotation angle and range of the parameter $z$ becomes $X_{m}(z)$.

(b) If $0<\phi \leq 2 \pi$, where $\phi$ is the rotation angle, we can utilize $T_{\text {rotation }}^{Z}$ to construct the first half and then utilize $\widetilde{T}_{\text {rotation }}^{Z}$ to construct the remaining according to the technique in $(a)$, with $(2 \pi-\phi) / \pi \leq z \leq 1$ value range of parameter $z$.

Proposition 3.9 From the reality of GBT-Bézier rotation surfaces which have various independent shape parameters, we can generate the desired shape of a rotation surface both locally and globally, by modifying shape control parameters. Moreover, translational and 


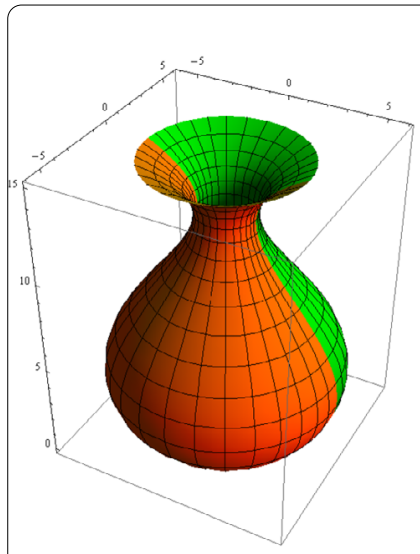

(a) $\mu=0, \nu=1$

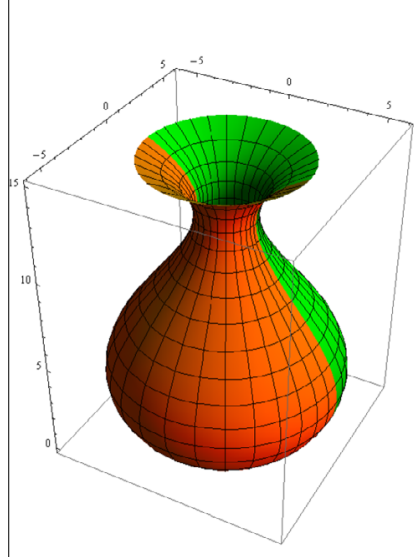

(d) $\mu=1, \nu=0$

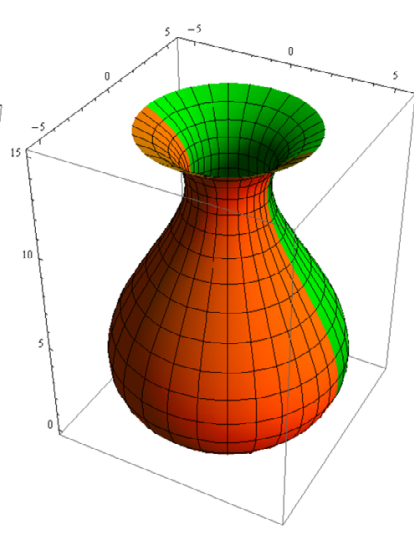

(b) $\mu=0, \nu=-1$

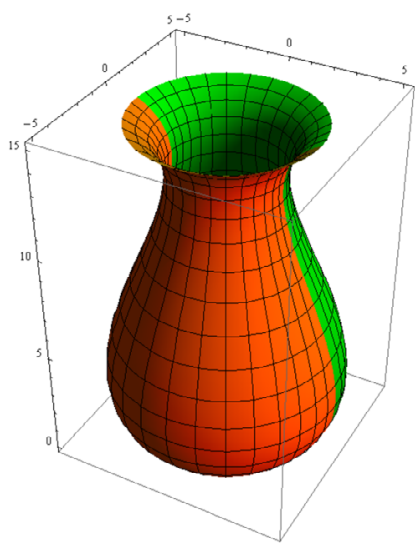

(e) $\mu, \nu=-1$

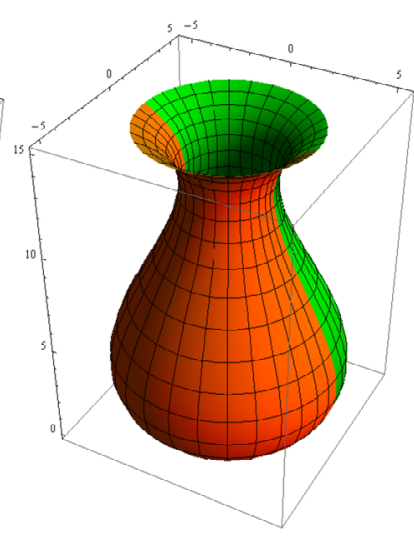

(c) $\mu=-1, \nu=0$

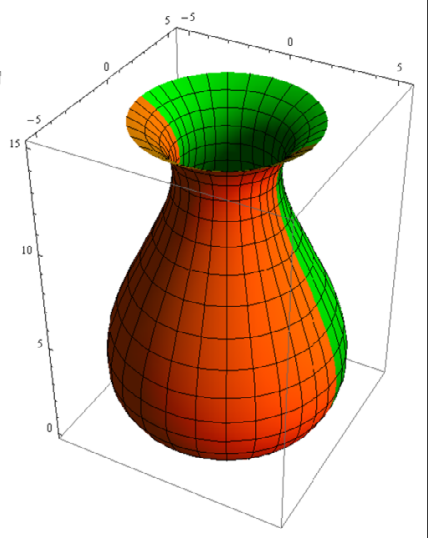

(f) $\mu=-0.3, \nu=-1$

Figure 21 GBT-Bézier rotation surfaces with a variety of shape control parameters

rotation transformation allows us to transfer the GBT-Bézier rotation surfaces in any direction to a particular position.

For constructing a GBT-Bézier rotation surface in the $X O Z$ plane, the detailed algorithm is as follows.

\section{Algorithm 6}

1. Take GBTB functions with shape parameters $\mu, v \in[-1,1]$.

2. Consider the control points $\left\{X_{k}, 0, Z_{k}\right\}(k=0,1, \ldots, m)$ in the $3 D$ plane.

3. Select the appropriate values of shape control parameters $\mu, v$ from the interval $[-1,1]$.

4. Calculate the functions $X_{m}(z)$ and $Z_{m}(z)$ by using expression (34).

5. Substitute the functions $X_{m}(z)$ and $Z_{m}(z)$ into expression (33) to achieve $T_{\text {rotation }}^{Z}\left(z, z_{1}, \mu, v\right)$ and $\widetilde{T}_{\text {rotation }}^{Z}\left(z, z_{1}, \mu, v\right)$ rotation surfaces.

6. Plot these two functions in Wolfram Mathematica software and join them to obtain GBT-Bézier rotation surfaces in the XOZ plane.

Example 3.6 Provided a variety of control points $Q_{l}(1=0,1,2,3,4)$ in the XOZ plane have the coordinates 


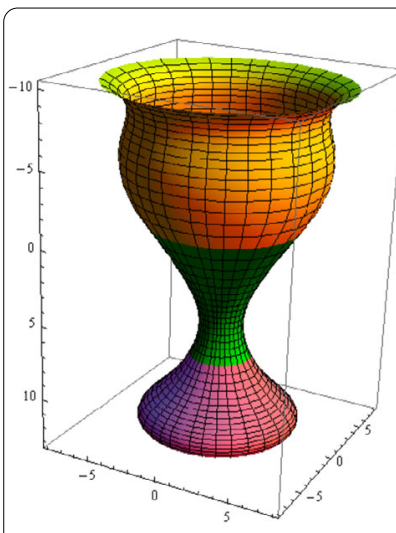

(a) $\mu, \nu, \mu_{1}, \nu_{1}, \mu_{2}, \nu_{2}=1$

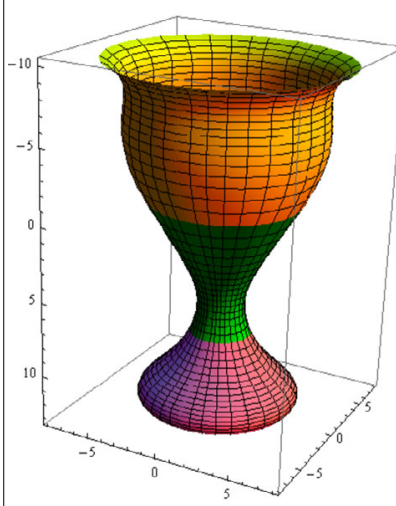

(d) $\mu, \nu=1, \mu_{1}, \nu_{1}=0.5, \mu_{2}, \nu_{2}=0.5$

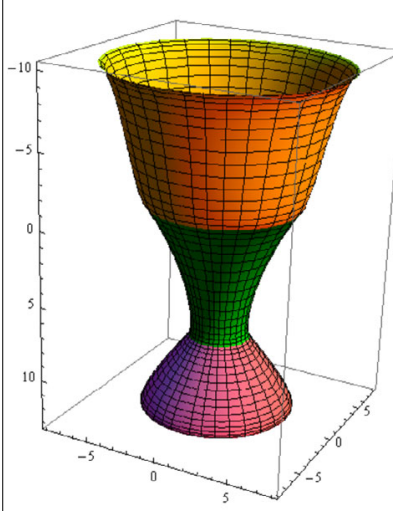

(g) $\mu, \nu, \mu_{1}, \nu_{1}, \mu_{2}, \nu_{2}=-1$

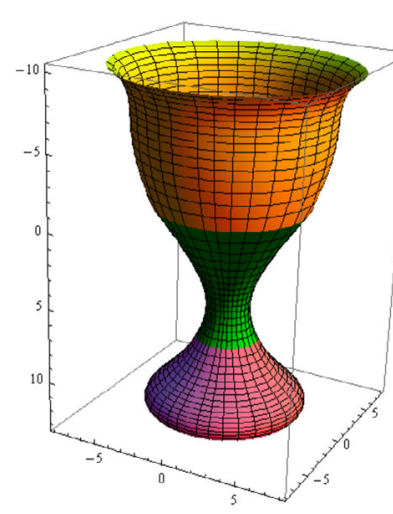

(b) $\mu, \nu, \mu_{1}, \nu_{1}=1, \mu_{2}, \nu_{2}=0$

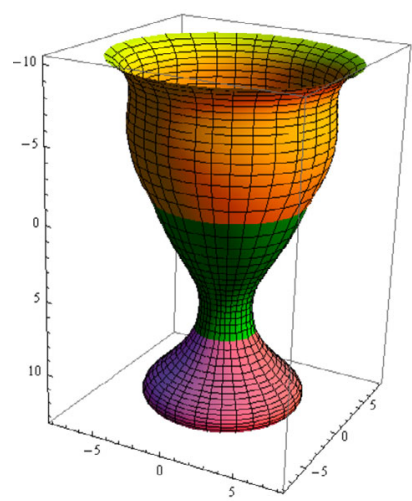

(e) $\mu, \nu=1, \mu_{1}, \nu_{1}=-0.3, \mu_{2}, \nu_{2}=0.5$

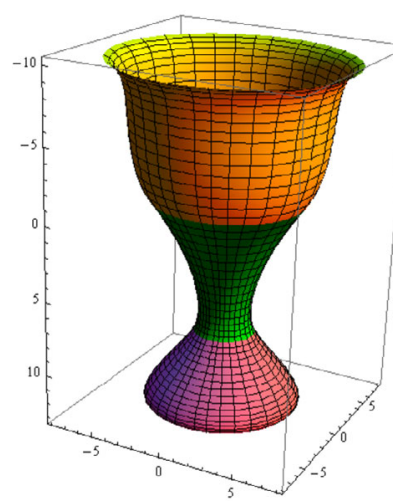

(h) $\mu, \nu, \mu_{1}, \nu_{1}, \mu_{2}, \nu_{2}=-0.2$

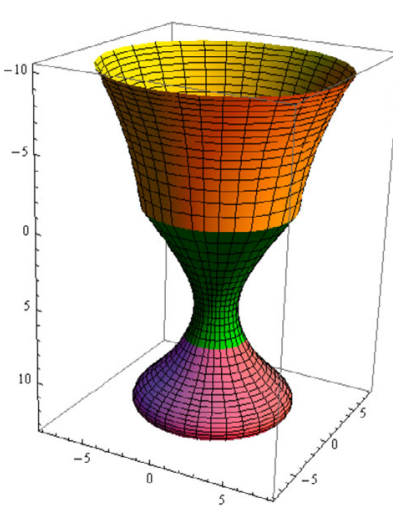

(c) $\mu, \nu, \mu_{1}, \nu_{1}=1, \mu_{2}, \nu_{2}=-1$

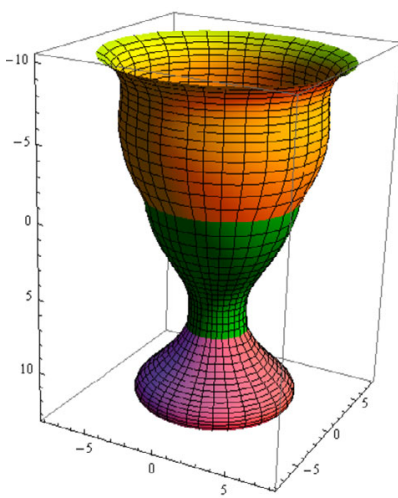

(f) $\mu, \nu=1, \mu_{1}, \nu_{1}=-1, \mu_{2}, \nu_{2}=0.5$

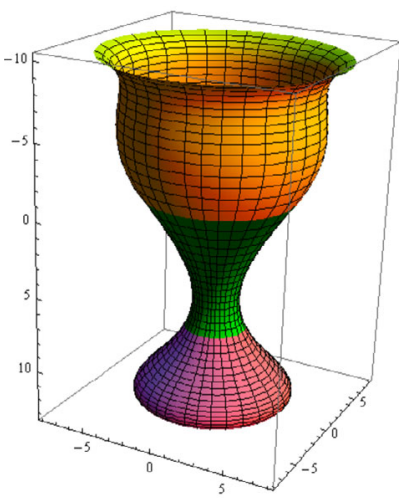

(i) $\mu, \nu, \mu_{1}, \nu_{1}, \mu_{2}, \nu_{2}=0.6$

Figure 22 Combine GBT-Bézier rotation surfaces with different shape parameters

$$
\left\{\begin{array}{l}
Q_{0}=(4,0,15), \\
Q_{1}=(0,0,13), \\
Q_{2}=(0,0,10), \\
Q_{3}=(10,0,5), \\
Q_{4}=(4,0,0) .
\end{array}\right.
$$




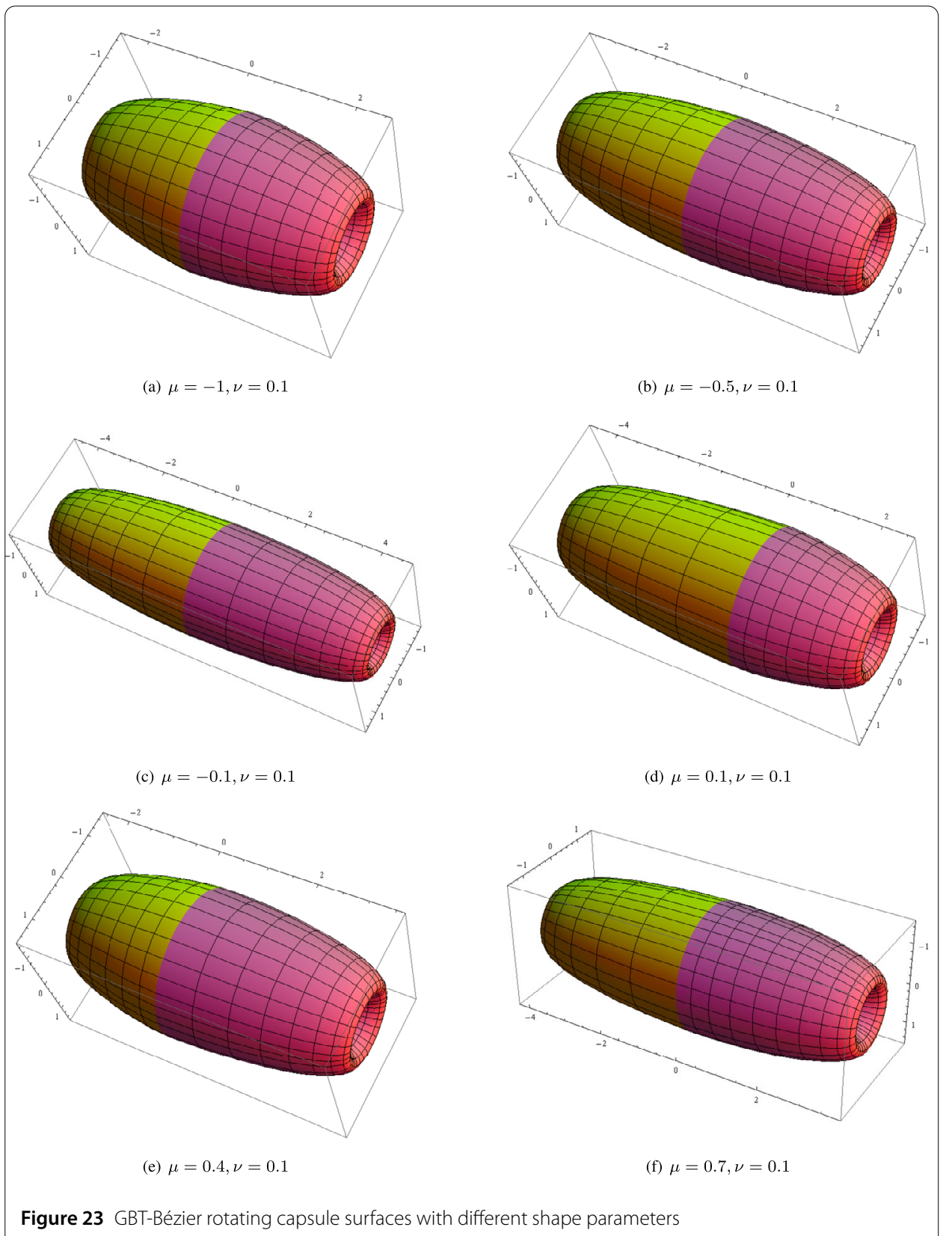

From equations (34) and (35), the parametric equation of GBT-Bézier curve $F_{4}(z, \mu, v)$ described by control points $Q_{i}(i=0,1,2,3,4)$ can be expressed as

$$
F_{4}(z, \mu, v)=\left\{X_{4}(z), 0, Z_{4}(z)\right\} \quad(0 \leq z \leq 1) .
$$

Hence, by using equations (33) and (35) and rotating the generating line $F_{4}(z, \mu, v)$ in one revolution around $z$-axis, the GBT-Bézier rotation surfaces are constructed as follows:

$$
\left\{\begin{array}{l}
T_{\text {rotation }}^{Z}\left(z, z_{1}, \mu, v\right)=\left\{\frac{1-2 z_{1}}{2 z_{1}^{2}-2 z_{1}+1} X_{4}(z), \frac{2 z_{1}-2 z_{1}^{2}}{2 z_{1}^{2}-2 z_{1}+1} X_{4}(z), Z_{4}(z)\right\} \\
\widetilde{T}_{\text {rotation }}^{Z}\left(z, z_{1}, \mu, v\right)=\left\{\frac{1-2 z_{1}}{2 z_{1}^{2}-2 z_{1}+1} X_{4}(z),-\frac{2 z_{1}-2 z_{1}^{2}}{2 z_{1}^{2}-2 z_{1}+1} X_{4}(z), Z_{4}(z)\right\}
\end{array}\right.
$$




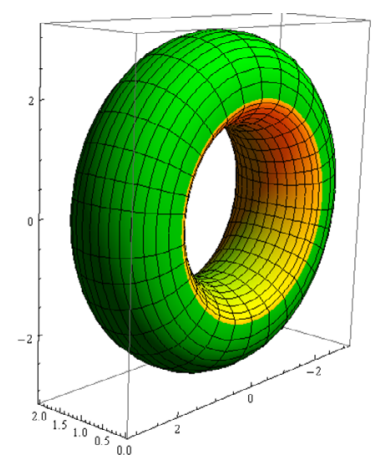

(a) $\mu=-1, \nu=0.1$

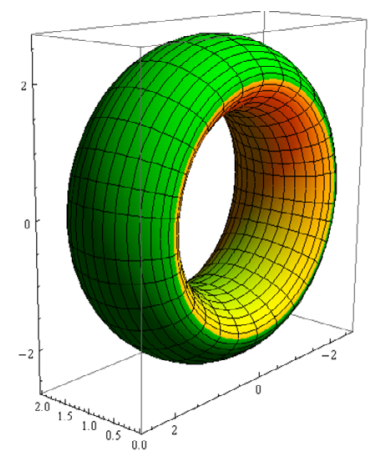

(c) $\mu=-0.1, \nu=0.1$

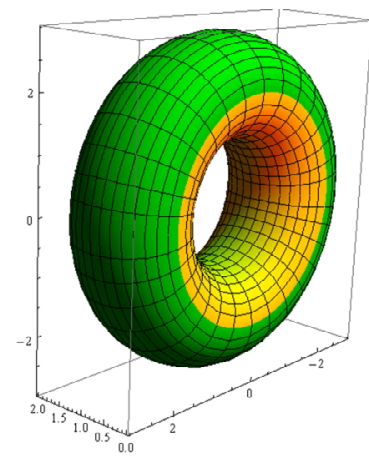

(e) $\mu=0.4, \nu=0.1$

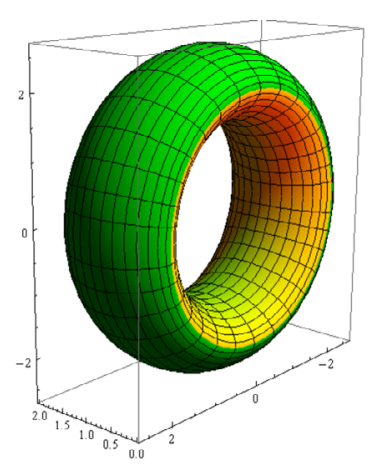

(b) $\mu=-0.5, \nu=0.1$

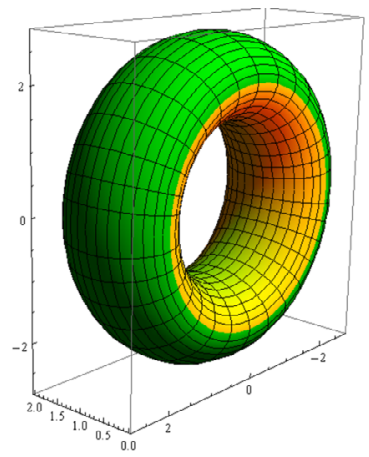

(d) $\mu=0.1, \nu=0.1$

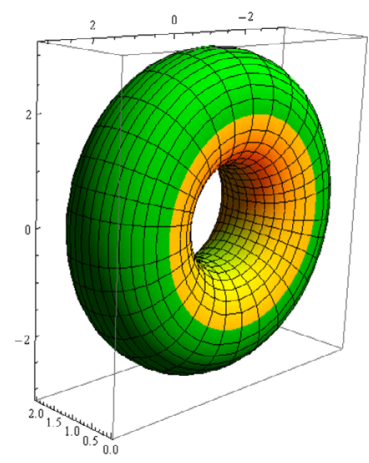

(f) $\mu=0.7, \nu=0.1$

Figure 24 GBT-Bézier rotating ring surfaces along various values of shape parameters

The graphs of GBT-Bézier rotation surfaces using control points (35) are presented in Fig. 21 with different shape parameters $\mu, v$ and with similar control polygon of generating lines.

Figures 22-27 also represent some GBT-Bézier rotation surfaces with different values of shape parameters and control polygon of generating lines.

\section{Conclusions}

This research work is mainly focused on the construction of engineering surfaces in Bézier approach. For this purpose, utilizing GBT-Bézier curves proposed in [24], six different 


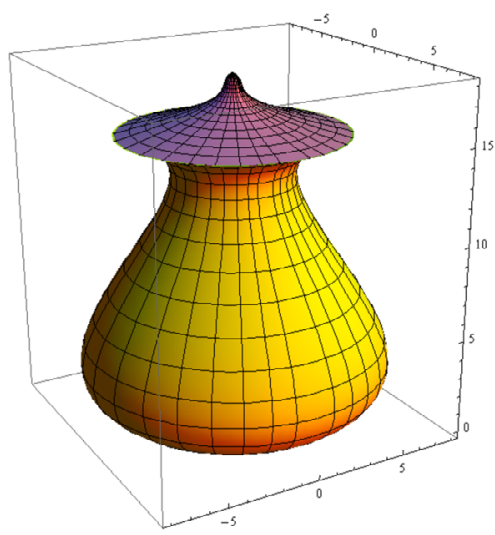

(a) $\mu=-1, \nu=0.1$

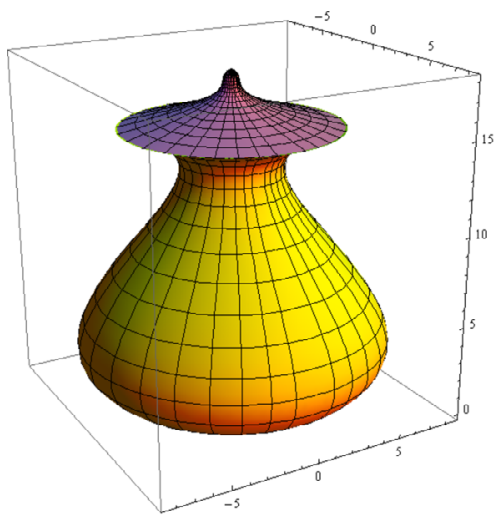

(c) $\mu=-0.1, \nu=0.1$

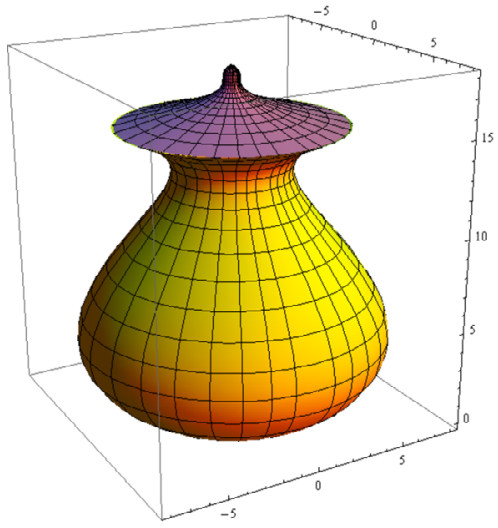

(e) $\mu=0.4, \nu=0.1$

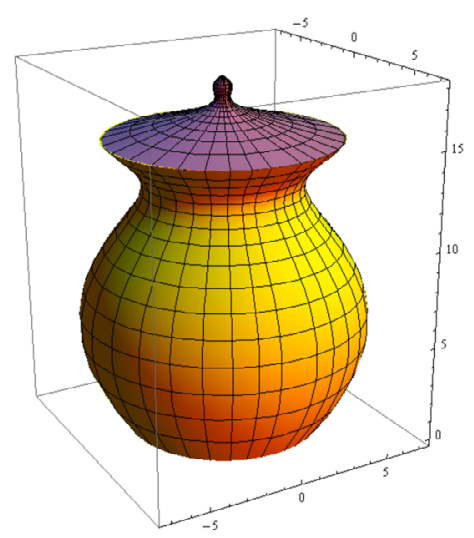

(b) $\mu=-0.5, \nu=0.1$

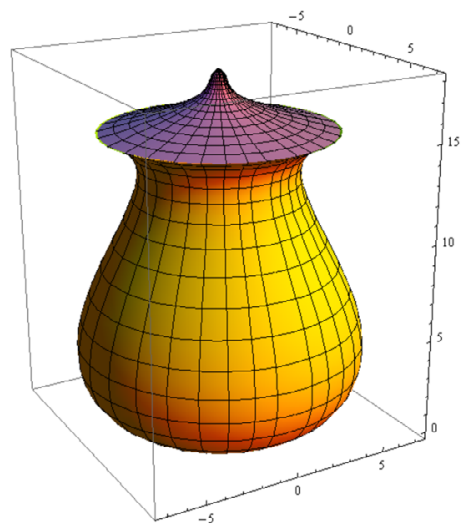

(d) $\mu=0.1, \nu=0.1$

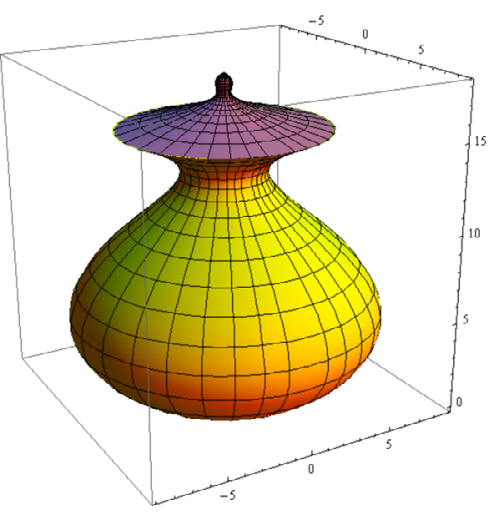

(f) $\mu=0.7, \nu=0.1$

Figure 25 Designing of GBT-Bézier rotating ceramic pot

types of tensor product GBT-Bézier engineering surfaces including GBT-Bézier cylinders, GBT-Bézier bilinear surfaces, GBT-Bézier ruled surfaces, GBT-Bézier swung surfaces, GBT-Bézier swept, and GBT-Bézier rotation surfaces are constructed. All geometric features of proposed GBT-Bézier surfaces are similar to the traditional Bézier, but the shape-adjustment feature is an additional feature that is not present in the traditional Bézier surface approach. The detailed algorithms for designing these GBT-Bézier engi- 


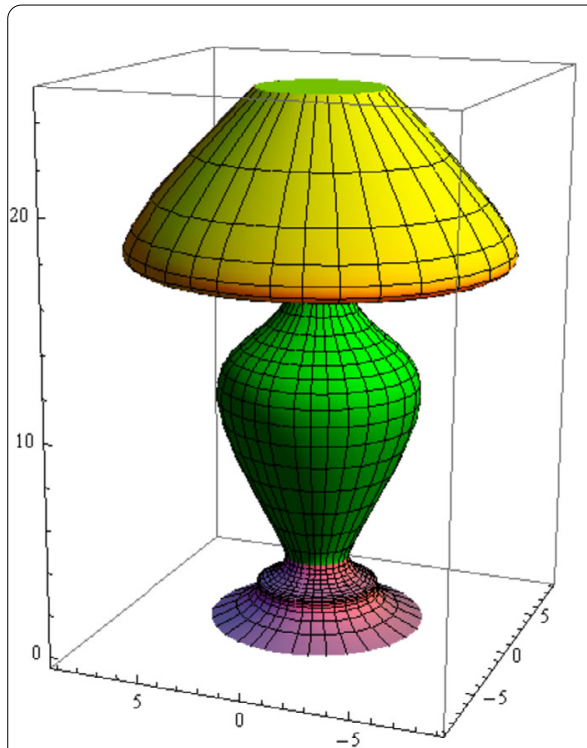

(a) $\forall \mu, \nu=1$

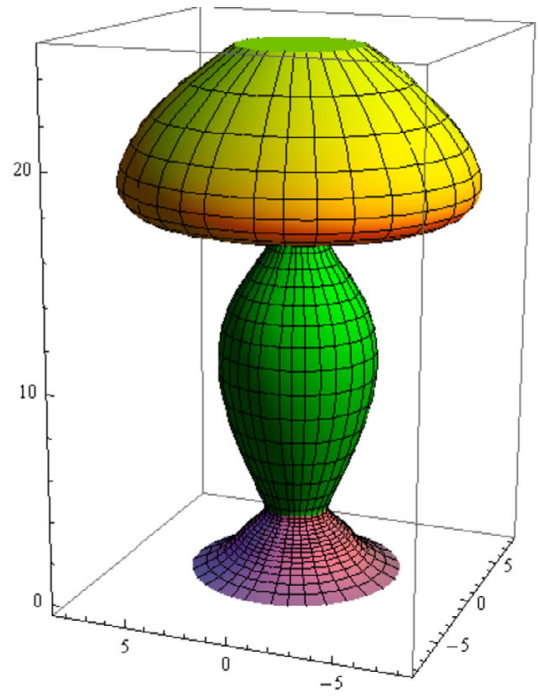

(c) $\mu, \mu_{1}, \nu, \nu_{1}=1, \mu_{2}, \mu_{3}, \nu_{2}, \nu_{3}=0$

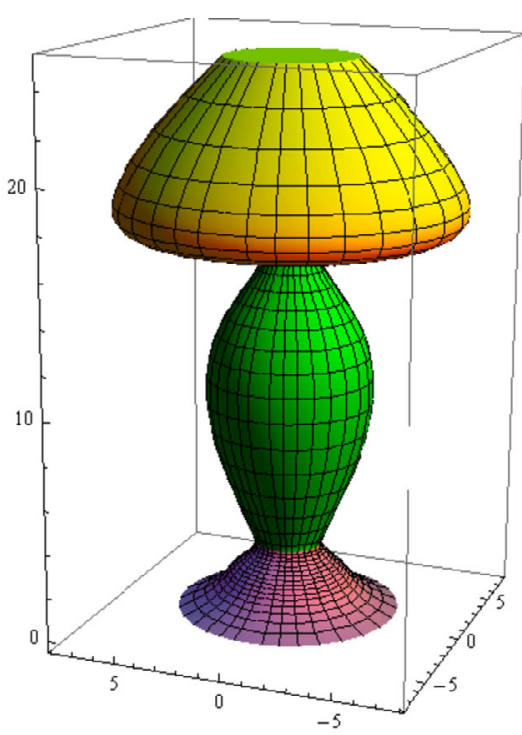

(b) $\forall \mu, \nu=-1$

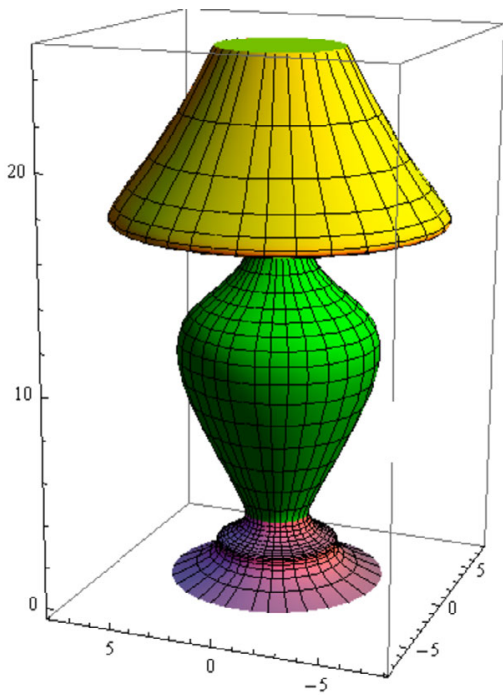

(d) $\mu, \mu_{1}, \nu, \nu_{1}=1, \mu_{2}, \mu_{3}, \nu_{2}, \nu_{3}=-1$

Figure $\mathbf{2 6}$ Design example of a lamp with different shape parameters

neering surfaces are also a part of this study. Furthermore, the construction of composite GBT-Bézier surfaces with $C^{1}, C^{2}$, and $C^{3}$ continuous connection is also presented. These special engineering surfaces not only have some parameters for their shape control but can also be expressed in terms of tensor product GBT-Bézier surface design (excluding rotation surfaces).

Some modeling samples of the proposed GBT-Bézier surfaces are presented here to illustrate that the designed GBT-Bézier surfaces can approach their convex hull better than the traditional Bézier surfaces approaches. Moreover, in the field of geometric modeling, these GBT-Bézier surfaces are very easy to implement. This study is meaningful and con- 


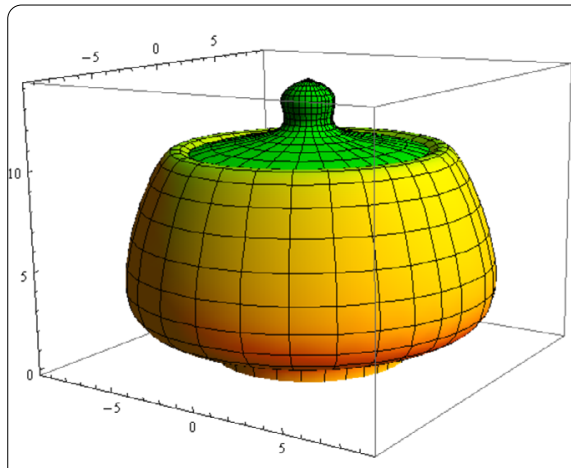

(a) $\forall \mu, \nu=1$

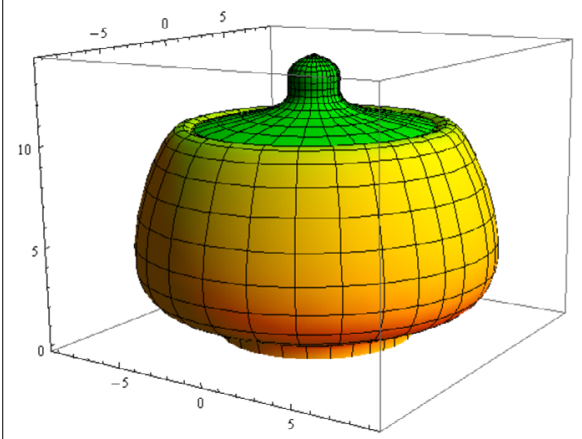

(c) $\forall \mu=0.95, \nu=-0.75$

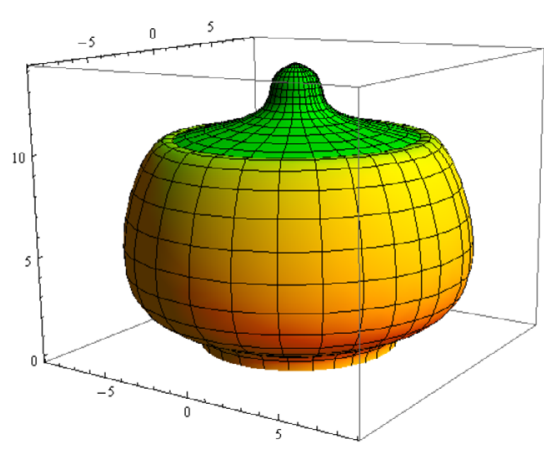

(b) $\forall \mu=-0.9, \nu=-0.85$

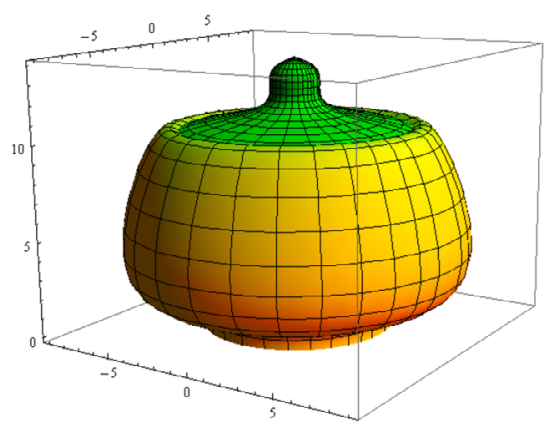

(d) $\mu=-0.8, \nu=0.9$

Figure $\mathbf{2 7}$ Modeling examples of a pot with different shape parameters

siderable in the sense that it will support us in modifying and constructing complicated engineering surfaces.

\section{Acknowledgements}

We thank Dr. Muhammad Amin for his assistance in proofreading the manuscript. This research was supported by the Department of Mechanical Engineering, Shizuoka University, Hamamatsu, 432-8561, Shizuoka, Japan.

\section{Funding}

No external funding is available for this research.

Availability of data and materials

Not applicable.

\section{Declarations}

Competing interests

The authors declare that they have no competing interests.

\section{Authors' contributions}

All authors equally contributed to this work. All authors read and approved the final manuscript.

\section{Author details}

${ }^{1}$ Department of Mathematics, University of Sargodha, Sargodha 40100, Pakistan. ${ }^{2}$ Department of Mechanical

Engineering, Shizuoka University, Hamamatsu, 432-8561, Shizuoka, Japan. ${ }^{3}$ Department of Mathematics, Division of Science and Technology, University of Education, Lahore 54770, Pakistan. ${ }^{4}$ School of Mathematical Sciences, Universiti Sains Malaysia, 11800 Penang, Malaysia.

\section{Publisher's Note}

Springer Nature remains neutral with regard to jurisdictional claims in published maps and institutional affiliations. 
Received: 17 August 2021 Accepted: 23 October 2021 Published online: 13 November 2021

\section{References}

1. Farin, G.E.: Curves and Surfaces for Computer-Aided Geometric Design: A Practical Guide. Academic Press, San Diego (2002)

2. Han, X., Ma, Y., Huang, X.: A novel generalization of Bézier curve and surface. J. Comput. Appl. Math. 217, 180-193 (2008)

3. Yang, L.Q., Zeng, X.M.: Bézier curves and surfaces with shape parameters. Int. J. Comput. Math. 86, 1253-1263 (2009)

4. Xiang, T., Liu, Z., Wang, W., Jiang, P.: A novel extension of Bézier curves and surfaces of the same degree. J. Inf. Comput. Sci. 7, 2080-2089 (2010)

5. Liu, Z., Chen, X., Jiang, P.: A class of generalized Bézier curves and surfaces with multiple shape parameters. J. Comput.-Aided Des. Comput. Graph. 22, 838-844 (2010)

6. Qin, X., Hu, G., Zhang, N., Shen, X., Yang, Y.: A novel extension to the polynomial basis functions describing Bézier curves and surfaces of degree $n$ with multiple shape parameters. Appl. Math. Comput. 223, 1-16 (2013)

7. Hu, G., Qin, X., Ji, X., Wei, G., Zhang, S.: The construction of $\lambda \mu$-B-spline curves and its application to rotational surfaces. Appl. Math. Comput. 266, 194-211 (2015)

8. Yan, L.L., Liang, Q.F.: An extension of the Bézier model. Appl. Math. Comput. 218, 2863-2879 (2011)

9. Hu, G., Cao, H., Wang, X., Qin, X.: G² continuity conditions for generalized Bézier-like surfaces with multiple shape parameters. J. Inequal. Appl. 2017, 248 (2017)

10. Hu, G., Wei, G., Wu, J.: Shape-adjustable generalized Bézier rotation surfaces with multiple shape parameters. J. Results Math. 72, 1281-1313 (2017)

11. Hu, G., Wu, J., Qin, X.: A novel extension of the Bézier model and its applications to surface modeling. Adv. Eng. Softw. $125,27-54(2018)$

12. Schoenberg, J.: On trigonometric spline interpolation. J. Math. Mech. 13, 795-825 (1964)

13. Lyche, T., Winther, R.: A stable recurrence relation for trigonometric B-splines. J. Approx. Theory 25, 266-279 (1979)

14. Hoschek, J., Lasser, D.: Fundamentals of Computer Aided Geometric Design. AK Peters, Natick (1993). Schumaker, L.L., translator

15. Majeed, A., Faiza, Q.: New rational cubic trigonometric B-spline curves with two shape parameters. Comput. Appl. Math. 39(3), 1-24 (2020)

16. Hu, G., Song, W.J.: New method for constructing quartic quasi-Bézier rotation surfaces with multiple shape parameters. J. Xi'an Jiaotong Univ. 48(6), 74-109 (2014)

17. Yan, L.L.: Cubic trigonometric nonuniform spline curves and surfaces. J. Math. Probl. Eng. 2016, 1-9 (2016)

18. Sharma, R.: Quartic trigonometric Bézier curves and surfaces with shape parameters. Int. J. Innov. Res. Comput. Commun. Eng. 4, 7712-7717 (2016)

19. Sharma, R.: Quasi-quartic trigonometric Bézier curves and surfaces with shape parameters. Int. J. Innov. Res. Sci. Eng Technol. 5, 6333-6337 (2016)

20. Sharma, R.: A class of Bézier-type cubic trigonometric curves and surfaces with shape parameters. Int. J. Recent Sci. Res. 7, 6333-6337 (2016)

21. BiBi, S., Abbas, M., Misro, M.Y., Hu, G.: A novel approach of hybrid trigonometric Bézier curve to the modeling of symmetric revolutionary curves and symmetric rotation surfaces. IEEE Access 7, 165779-165792 (2019)

22. Maqsood, M., Abbas, M., Hu, G., Ramli, A., Miura, K.T.: A novel generalization of trigonometric Bézier curve and surface with shape parameters and its applications. J. Math. Probl. Eng. 2020, 1-25 (2020)

23. BiBi, S., Abbas, M., Miura, T.K., Misro, Y.M.: Geometric modeling of novel generalized hybrid trigonometric Bézier-like curve with shape parameters and its applications. Mathematics 8, 967 (2020)

24. Maqsood, S., Abbas, M., Miura, K.T., Majeed, A., lqbal, A.: Geometric modeling and applications of generalized blended trigonometric Bézier curves with shape parameters. Adv. Differ. Equ. 2020, 550 (2020)

25. Piegl, L., Tiller, W.: The NURBS Book, 2nd edn., pp. 340-348. Springer, New York (1997)

26. Fan, J.H., Luo, G.M., Lin, X., Wang, W.M.: Surface modeling with C-Bézier in engineering. J. Mech. Eng. 39, 89-92 (2003)

\section{Submit your manuscript to a SpringerOpen ${ }^{\circ}$ journal and benefit from:}

- Convenient online submission

- Rigorous peer review

- Open access: articles freely available online

- High visibility within the field

- Retaining the copyright to your article

Submit your next manuscript at $\boldsymbol{s p r i n g e r o p e n . c o m ~}$ 